\title{
Data report: X-ray analyses of bulk sediment in IODP Holes U1320A and U1324B, northern Gulf of Mexico'
}

\author{
Cédric M. John ${ }^{2,3}$ and Thierry Adatte 4
}

\section{Chapter contents}

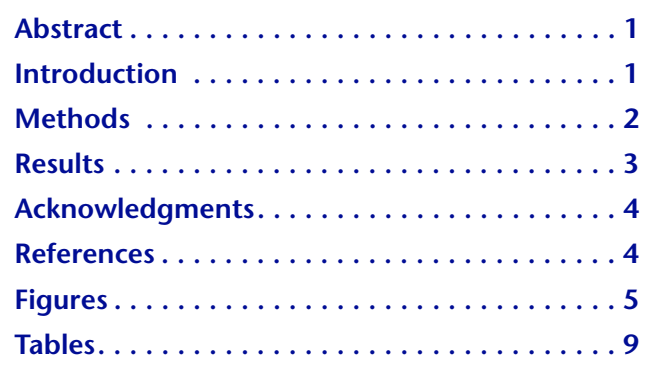

1John, C.M., and Adatte, T., 2009. Data report: Xray analyses of bulk sediment in IODP Holes U1320A and U1324B, northern Gulf of Mexico. In Flemings, P.B., Behrmann, J.H., John, C.M., and the Expedition 308 Scientists, Proc. IODP, 308: College Station, TX (Integrated Ocean Drilling Program Management International, Inc.). doi:10.2204/iodp.proc.308.214.2009

${ }^{2}$ Integrated Ocean Drilling Program, 1000

Discovery Drive, College Station TX 77845-9547, USA.

${ }^{3}$ Present address: Department of Earth Science and Engineering, Imperial College London, London SW7 2BP, United Kingdom.

cedric.john@imperial.ac.uk

${ }^{4}$ Institut de Géologie et Paléontologie, UNIL, CH1015 Lausanne, Switzerland.

\section{Abstract}

We present the results of $276 \mathrm{X}$-ray diffraction analyses of bulk sediment from Brazos-Trinity IV (Integrated Ocean Drilling Program [IODP] Hole U1320A) and Ursa (IODP Hole U1324B) Basins. The mineralogy at both locations is qualitatively very similar, with quartz and phyllosilicates dominating the assemblages and calcite, dolomite, K-feldspars, plagioclases, and halite present in varying amounts. Ankerite (iron-rich dolomite) was observed in only one sample. Although the composition of the sediment is relatively monotonous, trends in the percent occurrence of each fraction are observed and can be linked to changes in the lithostratigraphic units and core descriptions from Expedition 308. The data presented here will help establish a link between geomechanical behaviors of the sediments and matrix effects driven by mineral composition.

\section{Introduction}

Integrated Ocean Drilling Program (IODP) Expedition 308 drilled Pleistocene sediments from the Gulf of Mexico in Brazos-Trinity IV and Ursa Basins (see the "Expedition 308 summary" chapter). The goal of the expedition was to measure in situ pore pressure in a normally pressured (Brazos-Trinity IV) and an overpressured (Ursa) basin and compare these data with geotechnical measurements on the cores. During the expedition, a link was established between overpressured sediments and slope failure, leading to important turbidity transport and deposits (Flemings et al., 2008). Here, we present X-ray diffraction (XRD) data from bulk sediment from two holes, one from Brazos-Trinity IV Basin (Hole U1320A) and one from Ursa Basin (Hole U1324B). XRD is an excellent tool to quickly identify mineral phases in the sediment and quantify their abundances. The ultimate goal of our research is to tie the trends observed in the bulk rock mineralogy with similar trends in grain size (Sawyer et al.), in situ pore pressures (Flemings et al., 2008; Long et al.), and mechanical behavior of the sediment (Long et al.). Our working hypothesis is that the amount and type of clay minerals present in the matrix of the mudstone from both basins will likely affect sediment behavior. This integration, however, will require additional data, in particular detailed mineralogy of the phyllosilicate fraction (i.e., clay minerals), which is beyond the scope of this data report. 


\section{Methods}

A total of 276 samples were taken onboard the R/V JOIDES Resolution during Expedition 308 (77 samples from Hole U1320A and 199 samples from Hole U1324B). Sample volume was $10 \mathrm{~cm}^{3}$, and the sample frequency was 1 sample per $3 \mathrm{~m}$ (one every two core sections, typically taken in odd-numbered sections).

XRD analyses were carried out at the Geological Institute of the University of Neuchâtel, Switzerland. The samples were prepared following procedures described in Kübler (1987) and Adatte et al. (1996). Random powder of the bulk sample was used for characterization of the whole-rock mineralogy. Nearly 20 g of each sample was ground with a "jaw" crusher to obtain small chips (1-5 mm) of rock. Approximately $5 \mathrm{~g}$ was reserved for bulk sediment analyses and dried at $60^{\circ} \mathrm{C}$ and then ground again to a homogeneous powder with particle sizes $<40 \mu \mathrm{m}$. An aliquot of $800 \mathrm{mg}$ of this powder was pressed (20 bar) in a powder holder covered with a blotting paper and analyzed by XRD.

Whole-rock composition was determined by XRD (SCINTAG XRD 2000 diffractometer). The scans were done using the following conditions:

- X-ray powder diffractometer (SCINTAG XDS 2000)

- Spectral counter (THERMO ARL water-cooled silicon detector)

- Wavelength: $1.5406 \AA \mathrm{CuK \alpha} 1$

- Generator power: $45 \mathrm{kV}$ and $40 \mathrm{~mA}$

- Goniometer type: $\theta / \theta$

- Goniometer radius: $250 \mathrm{~mm}$

- Emitting slits: 2 and $4 \mathrm{~mm}$

- Receiving slits: 0.5 and $0.3 \mathrm{~mm}$

- Continuous scan

- Round (ø $25 \mathrm{~mm}$ ) glass plates

- Goniometer speed: $1 \% \mathrm{~min}$

- Acquisition step size: $0.03^{\circ}$

- Sample spinning: on

- Scanned interval: $2^{\circ}-65^{\circ} 2 \theta$

The files generated with SCINTAG are raw data (.RD) that are transformed in a routine by the software (DMS program, v. 2.63, graphic-normal display) into calculated (.NI) files. The calculations are fast fourier noise filter, background subtraction, and $\mathrm{K} \alpha 2$ stripping. The measurements are made on calculated files.

An internal standard (Kimmeridgian micritic limestone from the Ain region [France] composed of 99\% calcite) was run at the beginning of each day to track the aging of the X-ray source tube by looking at the intensity of the peak at $29.43^{\circ} 2 \theta$ and the data were corrected accordingly. The resulting diffractograms were analyzed using MacDiff 4.2.5, and each mineral in the assemblage was identified by the $h k l$ reflection of characteristic peaks (Table T1). The intensities of these characteristic peaks were collected for the semiquantitative analysis (Table T2). We chose peaks with no interference as much as possible, but this was not possible for plagioclase and K-feldspar, and we had to discriminate between their characteristic peaks by means of a profile-fitting function using MacDiff 4.2.5 and the Pearson VII method provided in this software.

Semiquantitative analysis was based on the following equation (Ferrero, 1965, 1966; Klug and Alexander, 1974; Kübler, 1983):

$$
I=I_{\mathrm{o}} \times C_{\mathrm{m}} \times\left(\mu_{\mathrm{m}} / \mu_{\mathrm{e}}\right),
$$

where

$I_{\mathrm{o}}=$ intensity of pure mineral (external standard, counts per second [cps]),

$I=$ intensity of the mineral to be quantified (cps),

$\mu_{\mathrm{m}}=$ mass absorption coefficient of pure mineral,

$\mu_{\mathrm{e}}=$ mass absorption coefficient of the sample (mineral + matrix), and

$C_{\mathrm{m}}=$ mineral concentration in the sample (wt\%).

The peak intensity $(I)$ of a mineral " $\mathrm{m}$ " in a given assemblage is a function of the abundance of " $\mathrm{m}$ " $\left(C_{\mathrm{m}}\right)$, the peak intensity (in counts per second) of an external standard containing $100 \%$ " $\mathrm{m}$ " $\left(I_{\mathrm{o}}\right)$, and the coefficient of mass absorption $\left(\mu_{\mathrm{m}}\right)$ of " $\mathrm{m}$ " as well as of the whole assemblage $\left(\mu_{\mathrm{e}}\right)$. This relationship is nonlinear, except when $\mu_{\mathrm{m}}=\mu_{\mathrm{e}}$. Determining $\mu_{\mathrm{e}}$ (or the coefficient of the so called "matrix") is the principal difficulty in using this technique, as it depends on the proportion of each mineral present in the matrix, which is unknown, as it is the quantity that we want to measure. Here, we follow the method of Ferrero (1966). We assume that the matrix is composed exclusively of clay minerals (micas, chlorites, kaolinites, and smectites), and we use a $\mu_{e}=47.0$, which was determined to be a good average value for a matrix dominated by illite in sedimentary rocks. We can then rewrite Equation 1 to calculate the weight percent of each mineral in the assemblage using

$$
C_{\mathrm{m}}=\left[\left(I \times \mu_{\mathrm{e}}\right) /\left(I_{\mathrm{o}} \times \mu_{\mathrm{m}}\right)\right] \times 100 .
$$

A list of the $100 \%$ intensity $\left(I_{0}\right)$ and coefficient of mass absorption $\left(\mu_{m}\right)$ of characteristic peaks for the external standards used is given in Table T1. This semiquantitative method leaves us with an "un- 
quantified" fraction of the rock matrix equals to $100-\Sigma C_{\mathrm{m}}$. This fraction averages $<5 \%$ but could be as high as $15 \%$ in some samples (Table T3). Two approaches with respect to the unquantified matrix can be considered. We can either assume that a portion of the rock matrix is either uncrystallized, poorly crystallized, or composed of phases present below detection limit and was thus not taken into account during the quantification process (this fraction could comprise organic matter or poorly crystallized minerals such as opal, phosphates, etc.) or we can assume that we underestimated the amount of phyllosilicates. Phyllosilicates represent a family of very different clay minerals (e.g., kaolinite, smectite, chlorite, etc.). We used the broad peak at $19.90^{\circ} 2 \theta$ as the characteristic peak for phyllosilicates, and we quantified them using a "standard" $\mu_{\mathrm{m}}$ of 47 (Table T1). But the specific mixing of these minerals present in our sediment is likely to deviate from the "average" composition estimated for $\mu_{\mathrm{m}}$, potentially explaining the amount of unquantified minerals. Both options are equally valid, and the correct quantification values are probably affected by a combination of both effects. Here, we decided to give the percent phyllosilicates values both with and without the added unquantified fraction (Figs. F1A, F2A; red curve represents weight percent phyllosilicates alone and the blue curve represents weight percent phyllosilicates with the unquantified fraction added). This gives a bracket for the "true" phyllosilicate content. In general, the difference between the two curves is small, giving us good confidence in our quantification results.

Instrumental error, caused by the fluctuation of the $\mathrm{X}$-ray source, detector, and amplification of the signal, has been estimated by measuring the same sample (100\% quartz) six times under the same conditions. The mean peak surface error is $<3 \%$ and the mean intensity error is $<1 \%$. Errors associated with the quantification step are more difficult to accurately predict as they depend on the actual matrix effect of each sample, which is unknown. Experience with this method suggests that a conservative error estimate for the quantified values would be 5\%-10\% for phyllosilicates and 5\% for grain minerals.

\section{Results}

The minerals identified (Table T2) are quartz, phyllosilicates, K-feldspars, plagioclases (sodic feldspar), calcite, dolomite, halite, and ankerite (Fe-rich dolomite, present as trace in a single sample [308U1320A-2H-5, 44-46 cm]). Phyllosilicates typically dominate the $<2 \mu \mathrm{m}$ fraction and are thus often called clay minerals, even though they can occur in a wide range of size fractions. Quartz, phyllosilicates, plagioclases, and feldspars are typical detrital minerals. Dolomite can either result from in situ diagenesis or (more likely in the levee deposits considered here) can be eroded from ancient carbonate rocks and subsequently transported by fluvial processes. Calcite typically comes from benthic and pelagic foraminifers such as the ones identified in Holes U1320A and U1324B (see the "Expedition 308 summary" chapter). Halite is most likely precipitated from pore water within the sediment, and so its concentration is a function of porosity and not of the primary sedimentologic composition of the rock.

\section{Stratigraphic evolution of mineralogy in Hole U1320A}

Lithostratigraphic Unit V (174.4-299.6 meters below seafloor [mbsf]) is characterized by an average of $23 \%$ quartz, 33\%-40\% phyllosilicates, 3\% K-feldspar, $6 \%$ plagioclases, $12 \%$ calcite, $14 \%$ dolomite, and $1 \%$ halite (Fig. F1A, F1B). The composition of this interval is very stable, although calcite and dolomite tend to decrease uphole of 200 mbsf in favor mostly of phyllosilicates.

Lithostratigraphic Unit IV (145.3-174.4 mbsf) is characterized by an average of 30\% quartz, 44\%-54\% phyllosilicates, 3\% K-feldspar, 5\% plagioclases, 3\% calcite, $4 \%$ dolomite, and 1\% halite (Fig. F1A, F1B). Compared to the previous intervals, carbonates are much less important and the lithology is dominated by thick sand units and turbidites (see the "Expedition 308 summary" chapter).

Lithostratigraphic Unit III (137.50-145.3 mbsf) is characterized by an average of $20 \%$ quartz, $42 \%-43 \%$ phyllosilicates, $15 \%$ K-feldspar, $4 \%$ plagioclases, 12\% calcite, $4 \%$ dolomite, and $1 \%$ halite (Fig. F1A, F1B). This interval contains the highest amount of K-feldspar found in this study and calcite values similar to Unit V. The lithology in this interval was interpreted as hemipelagic mudstones (see the "Expedition 308 summary" chapter).

Lithostratigraphic Unit II (2.65-137.50 mbsf) is characterized by an average of $47 \%$ quartz, 30\%-34\% phyllosilicates, $4 \% \mathrm{~K}$-feldspar, $4 \%$ plagioclases, $6 \%$ calcite, $4 \%$ dolomite, and 1\% halite (Fig. F1A, F1B). The mineralogical composition of this interval is variable, with quartz content ranging from $16 \%$ to $83 \%$. This variability reflects the interbedding of sandstone and mudstone identified during Expedition 308 (see the "Expedition 308 summary" chapter). No samples were analyzed from lithostratigraphic Unit I (upper $2.65 \mathrm{~m}$ of the hole). 


\section{Stratigraphic evolution of mineralogy in Hole U1324B}

Lithostratigraphic Unit II (364.70-600.80 mbsf) is characterized by an average of 33\% quartz, 27\%-30\% phyllosilicates, 7\% K-feldspar, 9\% plagioclases, $6 \%$ calcite, 14\% dolomite, and 1\% halite (Fig. F2A, F2B). The composition of this interval is very stable, although quartz content starts to decrease uphole of 400 mbsf in favor mostly of dolomite and, to a lesser extent, calcite. This interval was interpreted as overbank deposits (see the "Expedition 308 summary" chapter).

Lithostratigraphic Unit I (0-364.70 mbsf) is characterized by an average of 25\% quartz, 35\%-39\% phyllosilicates, 4\% K-feldspar, 6\% plagioclases, 20\% calcite, 15\% dolomite, and 1\% halite (Fig. F2A, F2B). Dolomite content is initially very high in this interval (average $=17 \%$; as much as $23 \%$ ), but trends show a steady decline in the content of this mineral starting at the base of Subunit IID (151.0 mbsf). From Subunit IC (107 mbsf) to the top of the hole, dolomite content averages $10 \%$ with a minimum of $5 \%$. This decrease in dolomite content is paralleled by an increase in calcite. The whole interval of Unit I was interpreted as a succession of rapidly deposited distal turbidite and intervals of discrete mass transport deposits (see the "Expedition 308 summary" chapter).

\section{Acknowledgments}

This research used samples and data provided by the Integrated Ocean Drilling Program (IODP). Funding for this research was provided by Joint Oceanographic Institutions, Inc./United States Science Support Program postcruise grant. We thank the captain, crew, technicians, and science party for their assis- tance onboard the JOIDES Resolution during IODP Expedition 308. Mike Underwood provided a thorough and constructive review that enhanced this data report.

\section{References}

Adatte, T., Stinnesbeck, W., and Keller, G., 1996. Lithostratigraphic and mineralogic correlations of near K/T boundary clastic sediments in northeastern Mexico: implications for origin and nature of deposition. Spec. Pap.-Geol. Soc. Am., 307:211-226.

Ferrero, J., 1965. Dosage des Principaux Minéraux des Roches par Diffraction de Rayon X: Bordeaux (Centre de la Formation en Petrolé).

Ferrero, J., 1966. Nouvelle Méthode Empirique pour le Dosage des Minéraux par Diffraction R.X.: Bordeaux (Centre de la Formation en Petrolé).

Flemings, P.B., Long, H., Dugan, B., Germaine, J., John, C.M., Behrmann, J.H., Sawyer, D., and IODP Expedition 308 Scientists, 2008. Pore pressure penetrometers document high overpressure near the seafloor where multiple submarine landslides have occurred on the continental slope, offshore Louisiana, Gulf of Mexico. Earth Planet. Sci. Lett., 269(3-4):309-325. doi:10.1016/ j.epsl.2007.12.005

Klug, H.P., and Alexander, L.E., 1974. X-ray Diffraction Procedures for Polycrystalline and Amorphous Materials: New York (Wiley and Sons).

Kübler, B., 1983. Dosage quantitatif des minéraux majeurs des roches sédimentaires par diffraction X. Cah. Inst. Geol. Neuchâtel, Ser. ADX, 1.

Kübler, B., 1987. Cristallinité de l'illite, méthodes normalisées de préparations, méthodes normalisées de mesures. Cah. Inst. Geol. Neuchâtel, Ser. ADX, 1.

Initial receipt: 22 August 2008

Acceptance: 15 April 2009

Publication: 29 June 2009

MS 308-214 
Figure F1. Stratigraphic evolution of mineralogy, Hole U1320A. Results are given in percent of the bulk sediment. A. Quartz, phyllosilicates, potassic feldspar (K-feldspar), and plagioclases (sodic plagioclases). Phyllosilicates: red curve = minimum quantification values without addition of the unquantified fraction, blue curve = maximum values with the added unquantified fraction (see the "Methods" chapter for details). (Continued on next page.)

A

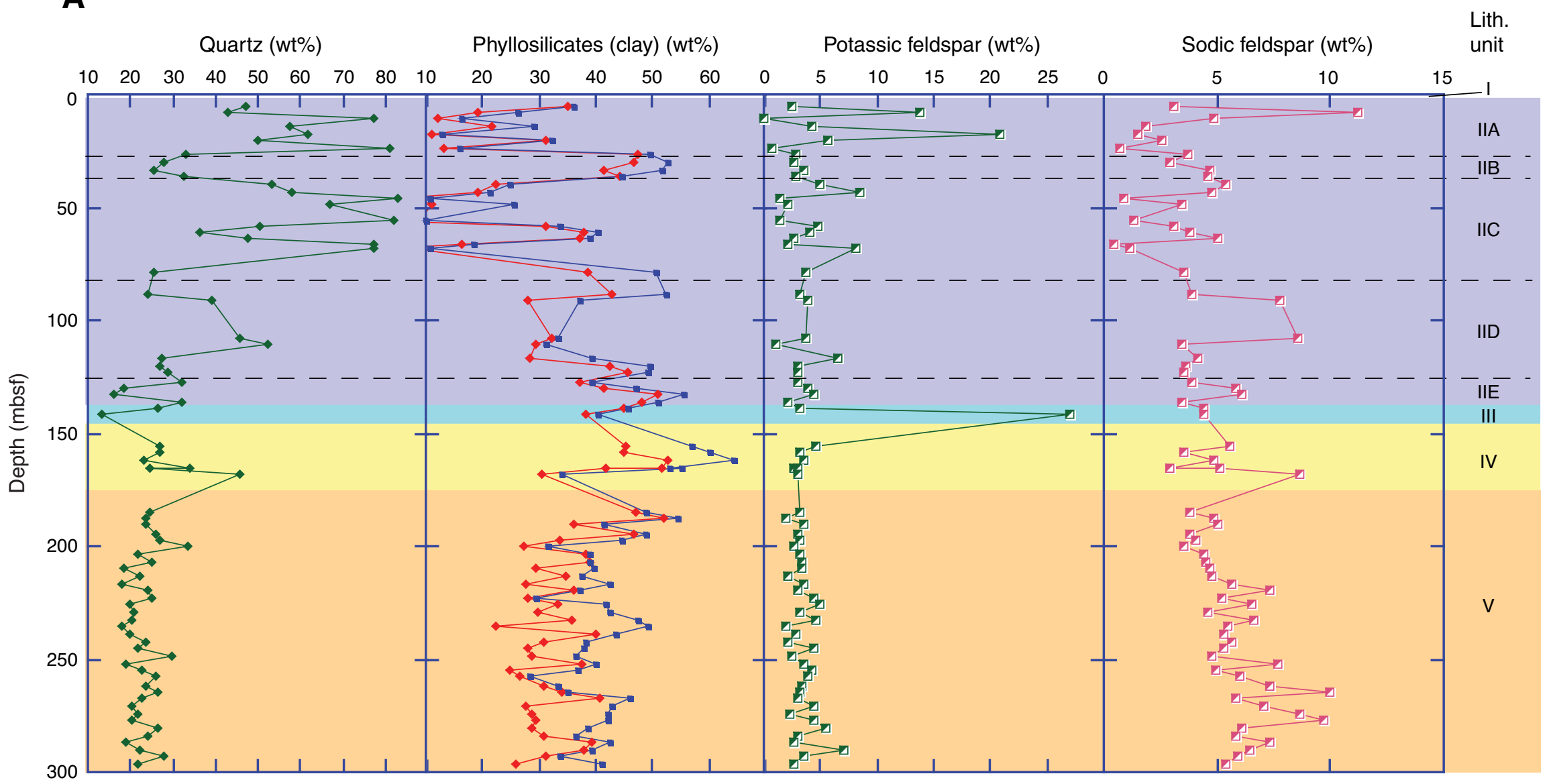




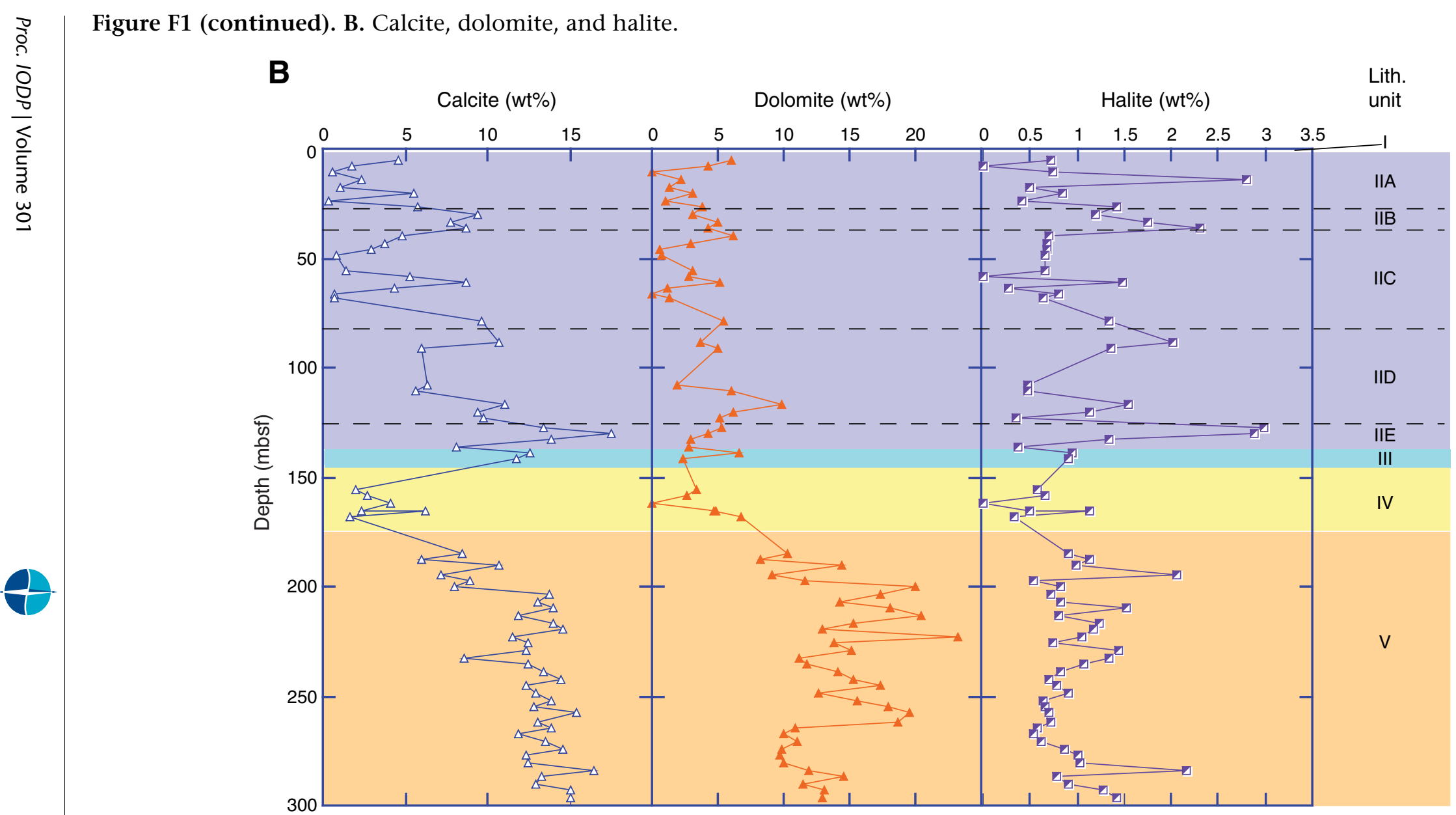


Figure F2. Stratigraphic evolution of mineralogy, Hole U1324B. Results are given in percent of the bulk sediment. A. Quartz, phyllosilicates, potassic feldspar (K-feldspar), and plagioclases (sodic plagioclases). Phyllosilicates: red curve = minimum quantification values without addition of the unquantified fraction, blue curve = maximum values with the added unquantified fraction (see the "Methods" chapter for details). (Continued on next page.)

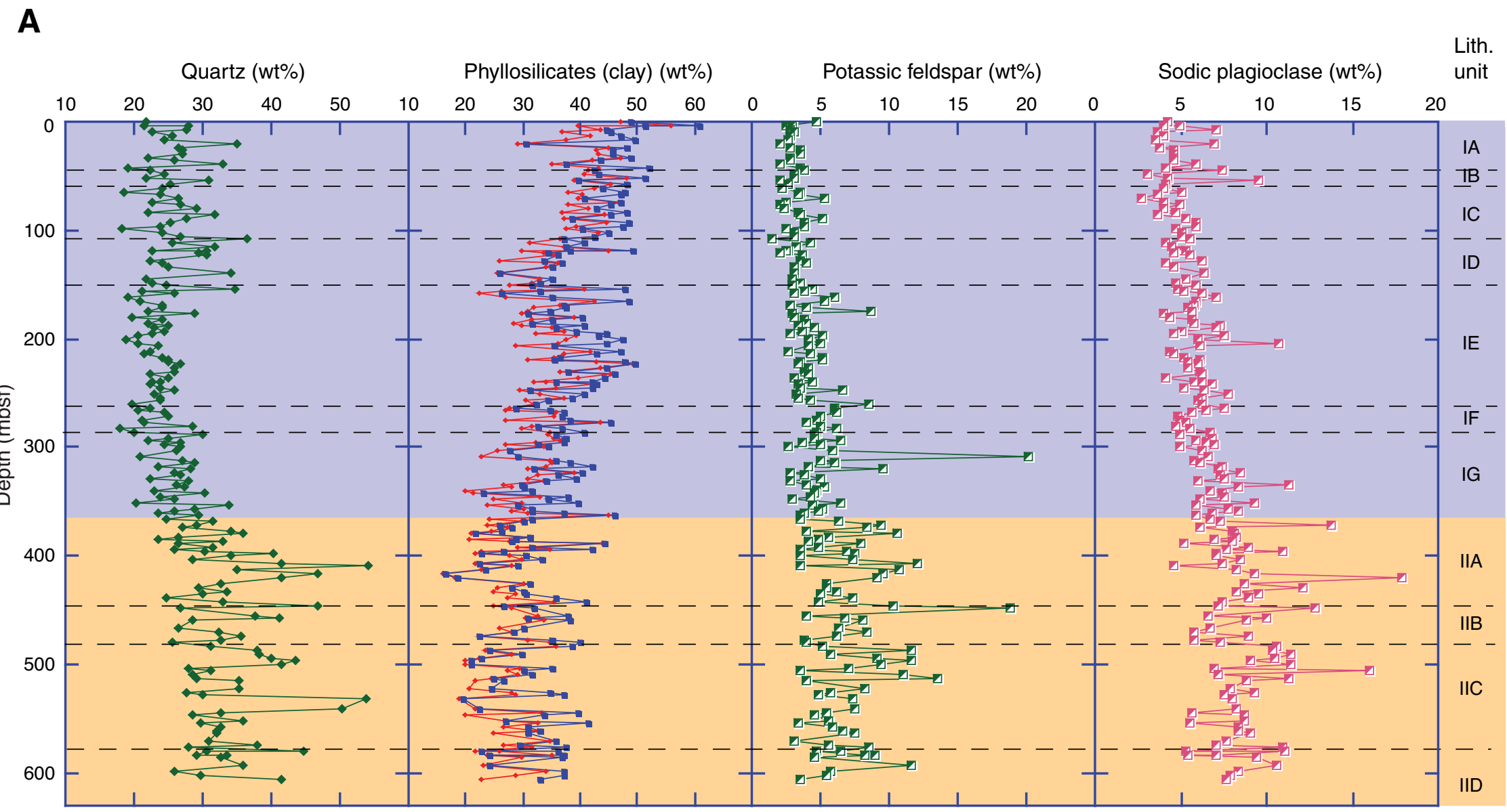


Figure F2 (continued). B. Calcite, dolomite, and halite.

B

Lith.

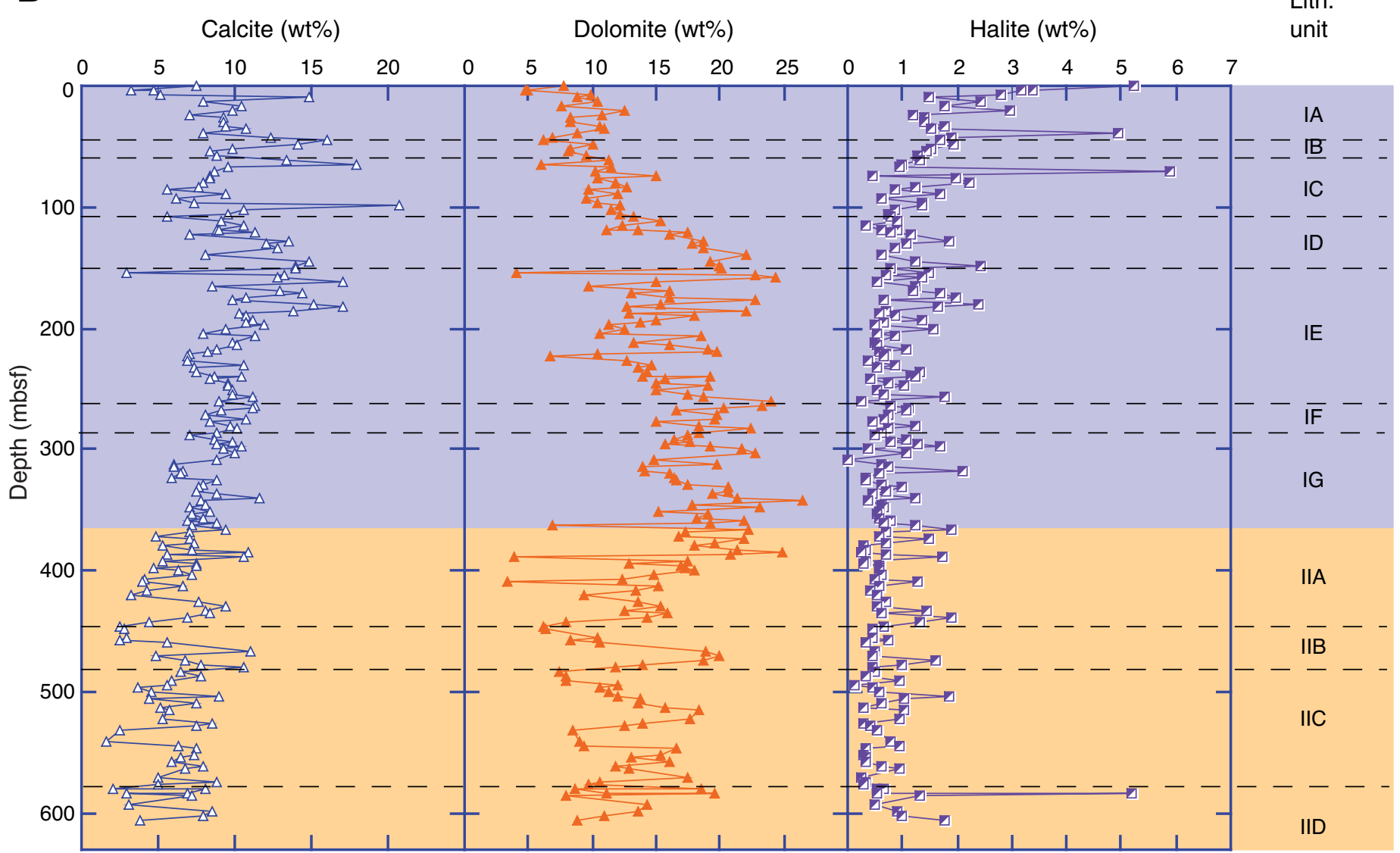


Table T1. Bulk rock XRD analyses data. (See table note.)

\begin{tabular}{lrrrrr}
\hline \multicolumn{1}{c}{ Mineral } & \multicolumn{1}{c}{$h k l$} & \multicolumn{1}{c}{$2 \theta$} & $\mathrm{d}(\AA)$ & \multicolumn{1}{c}{$I_{\mathrm{o}}(\mathrm{cpm})$} & \multicolumn{1}{c}{$\mu_{\mathrm{m}}$} \\
\hline Phyllosilicates & \multicolumn{1}{c}{-} & 19.900 & 4.460 & 20,000 & 47 \\
Quartz & 101 & 26.652 & 3.342 & 679,420 & 36 \\
Potassic feldspar & 2 & 27.507 & 3.240 & 72,550 & 51 \\
Sodic feldspar & 2 & 27.893 & 3.196 & 129,850 & 34 \\
Calcite & 104 & 29.406 & 3.035 & 231,380 & 74 \\
Ankerite & 104 & 30.797 & 2.901 & 346,037 & 108 \\
Dolomite & 104 & 30.939 & 2.888 & 316,840 & 49 \\
Halite & 200 & 31.693 & 2.821 & 487,807 & 78 \\
\hline
\end{tabular}

Note: $\mathrm{cpm}=$ counts per minute . 
Table T2. Characteristic peak analyses. (Continued on next four pages.) (See table note.)

\begin{tabular}{|c|c|c|c|c|c|c|c|c|c|}
\hline \multirow{2}{*}{$\begin{array}{l}\text { Core, section, } \\
\text { interval }\end{array}$} & \multirow{2}{*}{$\begin{array}{l}\text { Depth } \\
\text { (mbsf) }\end{array}$} & \multicolumn{8}{|c|}{ Peak (cps) } \\
\hline & & Phyllosilicates & Quartz & K-feldspar & Plagioclase & Calcite & Dolomite & Ankerite & Halite \\
\hline \multicolumn{10}{|l|}{ 308-U1320A- } \\
\hline $2 \mathrm{H}-1,44-46$ & 4.94 & 98 & 4,809 & 69 & 117 & 249 & 298 & 0 & 75 \\
\hline $2 \mathrm{H}-3,44-46$ & 7.94 & 24 & 3,155 & 286 & 288 & 30 & 95 & 0 & 0 \\
\hline $2 \mathrm{H}-5,44-46$ & 10.94 & 34 & 6,761 & 0 & 166 & 33 & 16 & 0 & 85 \\
\hline $3 \mathrm{H}-1,44-46$ & 14.44 & 42 & 4,320 & 76 & 26 & 65 & 53 & 0 & 291 \\
\hline $3 \mathrm{H}-3,60-62$ & 17.6 & 33 & 6,788 & 731 & 49 & 52 & 60 & 0 & 55 \\
\hline $3 \mathrm{H}-5,45-47$ & 20.45 & 78 & 3,986 & 131 & 78 & 253 & 125 & 0 & 85 \\
\hline $4 \mathrm{H}-1,44-46$ & 23.94 & 47 & 17,281 & 21 & 29 & 24 & 57 & 0 & 59 \\
\hline $4 \mathrm{H}-3,44-46$ & 26.94 & 114 & 2,520 & 64 & 108 & 255 & 151 & 0 & 139 \\
\hline $4 \mathrm{H}-5,44-46$ & 29.94 & 104 & 1,893 & 57 & 83 & 396 & 116 & 0 & 112 \\
\hline $5 \mathrm{H}-1,46-48$ & 33.46 & 83 & 1,070 & 33 & 80 & 245 & 118 & 0 & 71 \\
\hline $5 \mathrm{H}-3,45-47$ & 36.45 & 113 & 2,602 & 64 & 157 & 419 & 202 & 0 & 219 \\
\hline $5 \mathrm{H}-5,46-48$ & 39.46 & 79 & 8,235 & 151 & 211 & 326 & 294 & 0 & 80 \\
\hline $6 \mathrm{H}-1,45-47$ & 42.95 & 62 & 6,472 & 263 & 156 & 186 & 134 & 0 & 73 \\
\hline $6 \mathrm{H}-3,44-47$ & 45.94 & 32 & 9,061 & 65 & 66 & 207 & 76 & 0 & 78 \\
\hline $6 \mathrm{H}-5,45-47$ & 48.95 & 22 & 4,952 & 37 & 97 & 41 & 33 & 0 & 72 \\
\hline $7 \mathrm{H}-1,44-47$ & 52.44 & - & - & - & - & - & - & - & - \\
\hline 7H-3,44-47 & 55.44 & 29 & 8,169 & 35 & 33 & 74 & 145 & 0 & 77 \\
\hline $7 \mathrm{H}-5,44-47$ & 58.44 & 84 & 4,195 & 115 & 96 & 244 & 115 & 0 & 0 \\
\hline $8 \mathrm{H}-1,45-47$ & 61.05 & 100 & 3,027 & 109 & 116 & 419 & 241 & 0 & 143 \\
\hline $8 \mathrm{H}-3,45-47$ & 64.05 & 102 & 4,734 & 89 & 189 & 257 & 91 & 0 & 27 \\
\hline $8 \mathrm{H}-5,45-47$ & 66.14 & 51 & 8,056 & 65 & 14 & 35 & 0 & 0 & 94 \\
\hline $9 \mathrm{H}-1,45-47$ & 67.75 & 21 & 6,642 & 211 & 38 & 33 & 62 & 0 & 71 \\
\hline $11 X-1,42-44$ & 78.72 & 78 & 1,375 & 37 & 71 & 282 & 94 & 0 & 69 \\
\hline $12 X-1,45-47$ & 88.35 & 92 & 1,600 & 47 & 79 & 366 & 101 & 0 & 153 \\
\hline $12 X-3,45-47$ & 91.35 & 47 & 2,737 & 67 & 204 & 179 & 121 & 0 & 36 \\
\hline $14 X-1,47-49$ & 107.57 & 89 & 4,522 & 91 & 299 & 303 & 83 & 0 & 50 \\
\hline $14 X-3,46-49$ & 110.56 & 80 & 4,377 & 27 & 106 & 262 & 252 & 0 & 49 \\
\hline $15 X-1,44-46$ & 117.14 & 62 & 1,258 & 97 & 87 & 379 & 333 & 0 & 96 \\
\hline $15 X-3,44-46$ & 120.14 & 88 & 1,233 & 44 & 74 & 355 & 195 & 0 & 106 \\
\hline $15 X-5,44-46$ & 123.14 & 94 & 1,645 & 45 & 98 & 413 & 196 & 0 & 32 \\
\hline $16 X-1,107-109$ & 127.37 & 86 & 2,433 & 64 & 107 & 556 & 199 & 0 & 275 \\
\hline $16 X-3,45-47$ & 129.75 & 85 & 1,167 & 78 & 152 & 695 & 151 & 0 & 253 \\
\hline $16 X-5,41-43$ & 132.71 & 109 & 973 & 68 & 138 & 519 & 111 & 0 & 120 \\
\hline $17 X-1,41-43$ & 136.31 & 121 & 2,500 & 48 & 99 & 357 & 112 & 0 & 37 \\
\hline $17 X-3,45-47$ & 138.65 & 104 & 1,984 & 68 & 121 & 601 & 246 & 0 & 86 \\
\hline $17 X-5,44-46$ & 141.64 & 86 & 983 & 576 & 118 & 521 & 86 & 0 & 81 \\
\hline $19 X-1,45-47$ & 155.65 & 98 & 1,576 & 61 & 104 & 42 & 96 & 0 & 57 \\
\hline $19 X-3,45-47$ & 158.65 & 93 & 1,404 & 50 & 75 & 30 & 57 & 0 & 65 \\
\hline $19 X-5,45-47$ & 161.65 & 127 & 1,553 & 56 & 114 & 137 & 0 & 0 & 0 \\
\hline $20 X-1,46-48$ & 165.26 & 136 & 2,967 & 84 & 121 & 106 & 243 & 0 & 50 \\
\hline $20 X-1,46-48$ & 165.26 & 104 & 1,610 & 45 & 118 & 224 & 183 & 0 & 109 \\
\hline $20 X-3,44-46$ & 168.24 & 80 & 3,802 & 75 & 274 & 81 & 292 & 0 & 34 \\
\hline $22 \mathrm{X}-1,44-46$ & 184.54 & 115 & 1,885 & 71 & 108 & 361 & 397 & 0 & 86 \\
\hline $22 X-3,42-44$ & 187.52 & 123 & 1,764 & 43 & 139 & 261 & 324 & 0 & 109 \\
\hline $22 X-5,44-46$ & 190.54 & 72 & 1,533 & 53 & 113 & 410 & 508 & 0 & 91 \\
\hline $23 X-1,47-49$ & 194.27 & 115 & 2,099 & 71 & 132 & 329 & 394 & 0 & 196 \\
\hline $23 X-3,47-49$ & 197.27 & 79 & 1,844 & 50 & 88 & 301 & 373 & 0 & 51 \\
\hline $23 X-5,47-49$ & 200.27 & 62 & 2,451 & 57 & 100 & 347 & 733 & 0 & 80 \\
\hline $24 X-1,42-44$ & 203.82 & 86 & 1,537 & 66 & 121 & 567 & 639 & 0 & 66 \\
\hline $24 X-3,41-43$ & 206.81 & 88 & 1,805 & 73 & 123 & 543 & 533 & 0 & 75 \\
\hline $24 X-5,41-43$ & 209.81 & 55 & 1,098 & 71 & 129 & 442 & 572 & 0 & 92 \\
\hline $25 X-1,44-47$ & 213.44 & 89 & 1,745 & 66 & 131 & 535 & 791 & 0 & 73 \\
\hline $25 X-3,47-48$ & 216.47 & 48 & 1,129 & 54 & 126 & 456 & 482 & 0 & 81 \\
\hline $25 X-5,41-43$ & 219.41 & 84 & 1,781 & 64 & 230 & 602 & 520 & 0 & 107 \\
\hline $26 X-1,45-47$ & 223.05 & 73 & 1,964 & 94 & 166 & 483 & 943 & 0 & 97 \\
\hline $26 X-3,46-48$ & 225.87 & 62 & 1,224 & 106 & 151 & 440 & 448 & 0 & 68 \\
\hline $26 X-5,44-46$ & 228.85 & 65 & 1,352 & 67 & 125 & 467 & 525 & 0 & 132 \\
\hline $27 X-1,44-46$ & 232.64 & 78 & 1,429 & 80 & 159 & 323 & 388 & 0 & 125 \\
\hline $27 X-3,44-46$ & 235.64 & 32 & 848 & 21 & 123 & 377 & 232 & 0 & 68 \\
\hline $27 X-5,44-46$ & 238.64 & 90 & 1,426 & 59 & 144 & 553 & 524 & 0 & 75 \\
\hline $28 \mathrm{X}-1,45-47$ & 242.35 & 65 & 1,501 & 44 & 155 & 512 & 452 & 0 & 64 \\
\hline $28 X-3,45-47$ & 245.35 & 59 & 1,446 & 96 & 147 & 431 & 536 & 0 & 73 \\
\hline $28 X-5,45-47$ & 248.35 & 61 & 1,983 & 53 & 132 & 503 & 439 & 0 & 84 \\
\hline $29 X-1,42-44$ & 251.92 & 89 & 1,407 & 74 & 207 & 613 & 647 & 0 & 58 \\
\hline $29 X-3,42-44$ & 254.92 & 49 & 1,480 & 90 & 137 & 487 & 628 & 0 & 61 \\
\hline $29 X-5,42-44$ & 257.92 & 61 & 1,917 & 83 & 181 & 678 & 751 & 0 & 63 \\
\hline $30 X-1,44-46$ & 261.54 & 71 & 1,772 & 70 & 201 & 579 & 705 & 0 & 67 \\
\hline
\end{tabular}


Table T2 (continued). (Continued on next page.)

\begin{tabular}{|c|c|c|c|c|c|c|c|c|c|}
\hline \multirow{2}{*}{$\begin{array}{l}\text { Core, section, } \\
\text { interval }\end{array}$} & \multirow{2}{*}{$\begin{array}{l}\text { Depth } \\
\text { (mbsf) }\end{array}$} & \multicolumn{8}{|c|}{ Peak (cps) } \\
\hline & & Phyllosilicates & Quartz & K-feldspar & Plagioclase & Calcite & Dolomite & Ankerite & Halite \\
\hline $30 \times-3,44-46$ & 264.54 & 78 & 1,922 & 69 & 278 & 582 & 414 & 0 & 54 \\
\hline $30 X-5,43-45$ & 267.53 & 91 & 1,569 & 65 & 162 & 499 & 374 & 0 & 49 \\
\hline $31 X-1,45-47$ & 271.15 & 50 & 1,328 & 72 & 168 & 478 & 375 & 0 & 56 \\
\hline $31 X-3,45-47$ & 274.15 & 55 & 1,391 & 47 & 209 & 566 & 321 & 0 & 80 \\
\hline $31 X-5,45-47$ & 277.15 & 59 & 1,257 & 70 & 242 & 437 & 314 & 0 & 55 \\
\hline $32 X-1,44-46$ & 280.74 & 68 & 1,988 & 118 & 167 & 559 & 375 & 0 & 94 \\
\hline $32 X-3,42-44$ & 283.72 & 59 & 1,540 & 64 & 157 & 626 & 367 & 0 & 194 \\
\hline $32 X-5,43-45$ & 286.73 & 89 & 1,352 & 57 & 199 & 547 & 501 & 0 & 71 \\
\hline $33 X-1,44-46$ & 290.34 & 92 & 1,660 & 154 & 207 & 615 & 497 & 0 & 83 \\
\hline $33 X-3,44-46$ & 293.34 & 68 & 1,906 & 74 & 160 & 618 & 486 & 0 & 116 \\
\hline $33 X-5,45-47$ & 296.35 & 44 & 1,260 & 47 & 147 & 513 & 404 & 0 & 95 \\
\hline \multicolumn{10}{|l|}{ 308-U1324B- } \\
\hline $1 \mathrm{H}-1,45-47$ & 0.45 & 129 & 1,914 & 101 & 117 & 348 & 357 & 0 & 566 \\
\hline $1 \mathrm{H}-3,45-47$ & 3.45 & 132 & 1,606 & 56 & 114 & 141 & 182 & 0 & 324 \\
\hline $2 \mathrm{H}-1,45-47$ & 4.25 & 80 & 1,483 & 40 & 110 & 162 & 153 & 0 & 208 \\
\hline $2 \mathrm{H}-3,45-47$ & 7.25 & 106 & 2,333 & 64 & 214 & 266 & 388 & 0 & 269 \\
\hline $2 \mathrm{H}-5,34-36$ & 10.14 & 80 & 1,411 & 45 & 97 & 566 & 273 & 0 & 135 \\
\hline $3 \mathrm{H}-1,44-46$ & 13.74 & 95 & 1,801 & 62 & 112 & 293 & 347 & 0 & 229 \\
\hline $3 \mathrm{H}-3,44-46$ & 16.74 & 82 & 1,575 & 57 & 99 & 348 & 207 & 0 & 166 \\
\hline $3 \mathrm{H}-5,45-47$ & 19.75 & 109 & 3,250 & 68 & 221 & 505 & 573 & 0 & 464 \\
\hline $4 \mathrm{H}-1,44-47$ & 23.24 & 103 & 1,885 & 38 & 109 & 303 & 414 & 0 & 114 \\
\hline $4 \mathrm{H}-3,44-47$ & 26.24 & 97 & 1,921 & 76 & 130 & 395 & 317 & 0 & 134 \\
\hline $4 \mathrm{H}-5,45-47$ & 29.25 & 97 & 1,921 & 76 & 130 & 395 & 317 & 0 & 134 \\
\hline $5 \mathrm{H}-1,45-47$ & 32.75 & 109 & 1,540 & 62 & 127 & 348 & 368 & 0 & 164 \\
\hline $5 \mathrm{H}-3,45-47$ & 35.75 & 104 & 1,880 & 61 & 125 & 482 & 484 & 0 & 190 \\
\hline $5 \mathrm{H}-5,45-47$ & 38.75 & 109 & 3,250 & 68 & 221 & 505 & 573 & 0 & 464 \\
\hline $6 \mathrm{H}-1,45-47$ & 42.25 & 84 & 1,090 & 58 & 99 & 427 & 183 & 0 & 81 \\
\hline $6 \mathrm{H}-3,45-47$ & 45.25 & 115 & 1,901 & 79 & 273 & 733 & 228 & 0 & 151 \\
\hline $6 \mathrm{H}-5,45-47$ & 48.25 & 95 & 1,739 & 86 & 109 & 579 & 371 & 0 & 176 \\
\hline $7 \mathrm{H}-1,45-47$ & 51.75 & 106 & 1,508 & 66 & 118 & 374 & 275 & 0 & 143 \\
\hline $7 \mathrm{H}-3,45-47$ & 54.76 & 109 & 2,596 & 70 & 476 & 531 & 439 & 0 & 138 \\
\hline $7 \mathrm{H}-5,45-47$ & 57.76 & 103 & 1,813 & 57 & 117 & 375 & 366 & 0 & 121 \\
\hline $8 \mathrm{H}-1,44-47$ & 61.24 & 97 & 1,748 & 48 & 108 & 559 & 427 & 0 & 119 \\
\hline $8 \mathrm{H}-3,45-47$ & 64.25 & 77 & 923 & 53 & 109 & 567 & 115 & 0 & 90 \\
\hline $8 \mathrm{H}-5,45-47$ & 67.25 & 91 & 1,655 & 72 & 104 & 359 & 399 & 0 & 90 \\
\hline $9 \mathrm{H}-1,44-46$ & 70.74 & 91 & 1,946 & 132 & 101 & 309 & 410 & 0 & 795 \\
\hline $9 \mathrm{H}-3,45-47$ & 73.75 & 130 & 1,909 & 75 & 138 & 436 & 717 & 0 & 101 \\
\hline $9 \mathrm{H}-5,45-47$ & 76.75 & 88 & 2,177 & 70 & 145 & 280 & 325 & 0 & 32 \\
\hline $10 \mathrm{H}-1,43-45$ & 80.23 & 104 & 2,281 & 73 & 140 & 384 & 571 & 0 & 209 \\
\hline $10 \mathrm{H}-3,43-45$ & 83.23 & 83 & 1,398 & 53 & 104 & 285 & 450 & 0 & 118 \\
\hline $10 \mathrm{H}-5,43-45$ & 86.23 & 117 & 2,544 & 98 & 133 & 331 & 498 & 0 & 82 \\
\hline $11 \mathrm{H}-1,39-41$ & 89.69 & 109 & 2,914 & 134 & 178 & 486 & 648 & 0 & 347 \\
\hline $11 \mathrm{H}-3,44-46$ & 92.74 & 102 & 1,784 & 63 & 172 & 225 & 336 & 0 & 58 \\
\hline $11 \mathrm{H}-5,45-47$ & 95.75 & 92 & 1,782 & 83 & 168 & 318 & 404 & 0 & 128 \\
\hline $12 \mathrm{H}-1,45-47$ & 99.25 & 82 & 1,251 & 52 & 149 & 767 & 407 & 0 & 119 \\
\hline $12 \mathrm{H}-3,45-47$ & 102.25 & 108 & 2,027 & 90 & 172 & 449 & 515 & 0 & 83 \\
\hline $12 \mathrm{H}-5,45-47$ & 105.25 & 109 & 2,093 & 67 & 142 & 471 & 614 & 0 & 68 \\
\hline $13 \mathrm{H}-1,40-42$ & 108.7 & 104 & 3,323 & 58 & 163 & 294 & 613 & 0 & 138 \\
\hline $13 \mathrm{H}-3,45-47$ & 111.75 & 58 & 1,584 & 93 & 116 & 346 & 507 & 0 & 84 \\
\hline $13 \mathrm{H}-5,44-46$ & 114.74 & 96 & 2,495 & 70 & 126 & 498 & 512 & 0 & 78 \\
\hline $14 \mathrm{H}-1,44-46$ & 118.24 & 102 & 1,593 & 37 & 111 & 337 & 387 & 0 & 58 \\
\hline $14 \mathrm{H}-2,45-47$ & 119.21 & 61 & 2,159 & 36 & 152 & 307 & 459 & 0 & 87 \\
\hline $14 \mathrm{H}-3,45-47$ & 120.07 & 99 & 2,787 & 80 & 182 & 530 & 820 & 0 & 76 \\
\hline $14 \mathrm{H}-5,45-47$ & 122.1 & 88 & 2,363 & 81 & 160 & 337 & 638 & 0 & 110 \\
\hline $15 \mathrm{H}-1,45-47$ & 127.75 & - & - & - & - & - & - & - & - \\
\hline $15 \mathrm{H}-3,45-47$ & 130.75 & 87 & 2,175 & 106 & 166 & 616 & 835 & 0 & 99 \\
\hline $15 \mathrm{H}-5,45-47$ & 133.75 & 77 & 1,795 & 86 & 164 & 532 & 704 & 0 & 78 \\
\hline $16 \mathrm{H}-1,43-45$ & 136.73 & 45 & 1,247 & 54 & 143 & 440 & 589 & 0 & 77 \\
\hline $16 \mathrm{H}-3,43-45$ & 139.23 & 68 & 2,959 & 102 & 228 & 490 & 1034 & 0 & 60 \\
\hline $16 \mathrm{H}-5,45-47$ & 141.68 & - & - & - & - & - & - & - & - \\
\hline $17 \mathrm{H}-1,45-47$ & 145.35 & 67 & 1,355 & 61 & 143 & 566 & 666 & 0 & 111 \\
\hline $17 \mathrm{H}-3,45-47$ & 148.35 & 70 & 1,592 & 73 & 128 & 569 & 727 & 0 & 217 \\
\hline $17 \mathrm{H}-5,45-47$ & 151.35 & 61 & 1,690 & 64 & 162 & 541 & 714 & 0 & 72 \\
\hline $18 \mathrm{H}-1,44-46$ & 154.14 & 95 & 2,473 & 77 & 117 & 42 & 82 & 0 & 41 \\
\hline $18 \mathrm{H}-2,46-48$ & 155.66 & 52 & 1,238 & 60 & 117 & 469 & 806 & 0 & 65 \\
\hline $18 \mathrm{H}-3,49-51$ & 157.19 & 49 & 1,801 & 66 & 171 & 491 & 878 & 0 & 126 \\
\hline $19 \mathrm{H}-1,44-46$ & 162.44 & 55 & 1,205 & 102 & 153 & 613 & 474 & 0 & 48 \\
\hline $19 \mathrm{H}-3,44-46$ & 165.44 & 92 & 1,475 & 94 & 151 & 323 & 295 & 0 & 118 \\
\hline
\end{tabular}


Table T2 (continued). (Continued on next page.)

\begin{tabular}{|c|c|c|c|c|c|c|c|c|c|}
\hline \multirow{2}{*}{$\begin{array}{l}\text { Core, section, } \\
\text { interval }\end{array}$} & \multirow{2}{*}{$\begin{array}{l}\text { Depth } \\
\text { (mbsf) }\end{array}$} & \multicolumn{8}{|c|}{ Peak (cps) } \\
\hline & & Phyllosilicates & Quartz & K-feldspar & Plagioclase & Calcite & Dolomite & Ankerite & Halite \\
\hline $19 \mathrm{H}-5,44-46$ & 168.44 & 86 & 1,800 & 79 & 156 & 571 & 634 & 0 & 108 \\
\hline $20 \mathrm{H}-1,45-47$ & 171.25 & 70 & 1,652 & 83 & 149 & 557 & 452 & 0 & 61 \\
\hline $20 \mathrm{H}-3,45-47$ & 174.25 & 63 & 1,425 & 184 & 129 & 406 & 564 & 0 & 106 \\
\hline $20 \mathrm{H}-5,45-47$ & 177.25 & 68 & 2,094 & 86 & 140 & 500 & 937 & 0 & 61 \\
\hline $21 \mathrm{H}-1,45-47$ & 179.45 & 101 & 1,754 & 102 & 178 & 723 & 664 & 0 & 212 \\
\hline $21 \mathrm{H}-3,45-47$ & 182.45 & 61 & 1,421 & 81 & 152 & 616 & 430 & 0 & 55 \\
\hline $21 \mathrm{H}-5,45-47$ & 185.45 & 64 & 1,612 & 79 & 154 & 550 & 810 & 0 & 63 \\
\hline $22 \mathrm{H}-1,45-47$ & 187.35 & 60 & 1,388 & 53 & 178 & 353 & 418 & 0 & 56 \\
\hline $22 \mathrm{H}-3,45-47$ & 190.35 & 84 & 1,760 & 104 & 192 & 570 & 786 & 0 & 79 \\
\hline $22 \mathrm{H}-5,45-47$ & 193.35 & 85 & 1,779 & 79 & 139 & 466 & 565 & 0 & 126 \\
\hline $23 \mathrm{H}-1,44-46$ & 194.24 & 74 & 1,659 & 60 & 129 & 451 & 522 & 0 & 60 \\
\hline $23 \mathrm{H}-3,45-47$ & 197.25 & 90 & 1,503 & 110 & 209 & 457 & 391 & 0 & 46 \\
\hline $24 \mathrm{H}-1,45-47$ & 200.85 & 82 & 1,309 & 66 & 139 & 359 & 441 & 0 & 50 \\
\hline $24 \mathrm{H}-3,45-47$ & 203.85 & 81 & 1,382 & 89 & 279 & 303 & 371 & 0 & 53 \\
\hline $24 \mathrm{H}-5,44-46$ & 206.84 & 57 & 1,513 & 89 & 170 & 394 & 644 & 0 & 82 \\
\hline $25 \mathrm{H}-1,44-46$ & 207.94 & - & - & - & - & - & - & - & - \\
\hline $25 \mathrm{H}-3,45-47$ & 210.95 & 94 & 1,569 & 56 & 124 & 376 & 466 & 0 & 46 \\
\hline $25 \mathrm{H}-5,45-47$ & 213.95 & 81 & 1,508 & 91 & 129 & 389 & 572 & 0 & 51 \\
\hline $26 \mathrm{H}-1,44-46$ & 216.54 & 82 & 1,772 & 113 & 146 & 376 & 781 & 0 & 101 \\
\hline $26 \mathrm{H}-3,45-47$ & 219.55 & 67 & 1,481 & 88 & 142 & 307 & 721 & 0 & 54 \\
\hline $26 \mathrm{H}-5,45-47$ & 222.05 & 103 & 1,867 & 79 & 154 & 308 & 408 & 0 & 68 \\
\hline $27 \mathrm{H}-1,45-47$ & 222.95 & 124 & 2,090 & 76 & 183 & 341 & 305 & 0 & 62 \\
\hline $27 \mathrm{H}-3,45-47$ & 225.95 & 103 & 1,934 & 90 & 154 & 339 & 538 & 0 & 36 \\
\hline $28 \mathrm{H}-1,44-46$ & 229.54 & 84 & 1,893 & 81 & 172 & 487 & 594 & 0 & 80 \\
\hline $28 \mathrm{H}-3,44-46$ & 232.56 & 113 & 1,806 & 89 & 177 & 358 & 566 & 0 & 49 \\
\hline $28 \mathrm{H}-5,44-46$ & 235.56 & 91 & 1,808 & 70 & 118 & 283 & 519 & 0 & 51 \\
\hline $29 \mathrm{H}-1,45-47$ & 239.05 & 55 & 1,313 & 73 & 134 & 323 & 494 & 0 & 110 \\
\hline $29 \mathrm{H}-2,45-47$ & 240.55 & 78 & 1,579 & 96 & 173 & 439 & 725 & 0 & 114 \\
\hline $29 \mathrm{H}-3,45-47$ & 242.05 & 105 & 1,879 & 74 & 193 & 436 & 676 & 0 & 40 \\
\hline $29 \mathrm{H}-5,45-47$ & 245.05 & 77 & 1,657 & 56 & 146 & 321 & 500 & 0 & 70 \\
\hline $30 \mathrm{H}-1,45-47$ & 247.75 & 71 & 1,961 & 143 & 179 & 447 & 769 & 0 & 95 \\
\hline $30 \mathrm{H}-3,45-47$ & 250.75 & 72 & 1,549 & 71 & 191 & 375 & 539 & 0 & 49 \\
\hline $30 \mathrm{H}-5,45-47$ & 253.75 & 86 & 1,755 & 73 & 174 & 418 & 669 & 0 & 61 \\
\hline $31 \mathrm{H}-1,45-47$ & 257.25 & 68 & 1,664 & 91 & 166 & 431 & 675 & 0 & 69 \\
\hline $31 \mathrm{H}-3,45-47$ & 260.25 & 74 & 1,506 & 184 & 173 & 378 & 982 & 0 & 69 \\
\hline $31 \mathrm{H}-5,45-47$ & 263.25 & 63 & 1,617 & 130 & 207 & 475 & 880 & 0 & 72 \\
\hline $32 \mathrm{H}-1,45-47$ & 265.05 & 60 & 1,274 & 108 & 153 & 306 & 619 & 0 & 104 \\
\hline $32 \mathrm{H}-3,45-47$ & 268.05 & 85 & 1,853 & 133 & 157 & 389 & 668 & 0 & 102 \\
\hline $32 \mathrm{H}-5,45-47$ & 271.05 & 89 & 1,881 & 106 & 130 & 356 & 776 & 0 & 69 \\
\hline $33 \mathrm{H}-1,43-45$ & 274.13 & 60 & 1,476 & 80 & 112 & 323 & 671 & 0 & 62 \\
\hline $33 \mathrm{H}-3,45-47$ & 277.05 & 113 & 1,790 & 107 & 177 & 399 & 617 & 0 & 42 \\
\hline $33 \mathrm{H}-5,44-46$ & 280.04 & 74 & 2,146 & 130 & 160 & 451 & 740 & 0 & 116 \\
\hline $34 \mathrm{H}-1,45-47$ & 282.95 & 63 & 1,230 & 132 & 153 & 382 & 805 & 0 & 69 \\
\hline $34 \mathrm{H}-3,45-47$ & 285.95 & 79 & 1,417 & 104 & 191 & 291 & 594 & 0 & 60 \\
\hline $34 \mathrm{H}-5,45-47$ & 288.95 & 82 & 2,268 & 101 & 141 & 308 & 685 & 0 & 48 \\
\hline $35 \mathrm{H}-1,45-47$ & 290.85 & 81 & 1,781 & 90 & 186 & 331 & 589 & 0 & 104 \\
\hline $35 \mathrm{H}-3,45-47$ & 293.85 & 84 & 1,613 & 140 & 163 & 414 & 671 & 0 & 73 \\
\hline $35 \mathrm{H}-4,44-47$ & 295.34 & 73 & 1,923 & 82 & 183 & 338 & 568 & 0 & 123 \\
\hline $36 \mathrm{H}-1,44-46$ & 296.84 & 60 & 1,727 & 108 & 197 & 396 & 692 & 0 & 64 \\
\hline $36 \mathrm{H}-3,47-49$ & 299.87 & 83 & 2,127 & 79 & 168 & 436 & 909 & 0 & 36 \\
\hline $36 \mathrm{H}-5,44-46$ & 302.84 & 59 & 1,930 & 127 & 174 & 424 & 866 & 0 & 100 \\
\hline $37 \mathrm{H}-3,45-48$ & 309.05 & 48 & 1,453 & 435 & 147 & 331 & 525 & 0 & 0 \\
\hline $38 \mathrm{H}-1,45-47$ & 311.75 & 85 & 2,073 & 111 & 166 & 302 & 814 & 0 & 59 \\
\hline $38 \mathrm{H}-3,45-47$ & 314.75 & 81 & 2,106 & 112 & 148 & 264 & 551 & 0 & 71 \\
\hline $38 \mathrm{H}-5,45-47$ & 317.75 & 76 & 1,609 & 69 & 179 & 245 & 515 & 0 & 110 \\
\hline $39 \mathrm{H}-1,45-47$ & 319.65 & 75 & 2,167 & 231 & 227 & 323 & 668 & 0 & 54 \\
\hline $39 \mathrm{H}-3,45-47$ & 322.65 & 98 & 2,051 & 76 & 294 & 262 & 653 & 0 & 32 \\
\hline $39 \mathrm{H}-5,45-47$ & 325.53 & 72 & 1,871 & 86 & 210 & 318 & 598 & 0 & 33 \\
\hline $40 \mathrm{H}-1,44-46$ & 328.04 & 64 & 1,430 & 89 & 214 & 292 & 628 & 0 & 58 \\
\hline $40 \mathrm{H}-3,44-46$ & 331.04 & 89 & 2,546 & 103 & 239 & 418 & 882 & 0 & 93 \\
\hline $41 \mathrm{H}-1,44-46$ & 333.94 & 53 & 1,836 & 90 & 329 & 282 & 770 & 0 & 69 \\
\hline $41 \mathrm{H}-3,48-50$ & 336.98 & 64 & 1,974 & 116 & 211 & 340 & 711 & 0 & 42 \\
\hline $42 \mathrm{H}-1,44-46$ & 339.44 & 36 & 1,317 & 80 & 189 & 326 & 658 & 0 & 116 \\
\hline $42 \mathrm{H}-3,45-47$ & 342.45 & 62 & 2,296 & 101 & 214 & 339 & 1041 & 0 & 38 \\
\hline $43 \mathrm{H}-1,44-46$ & 345.44 & 71 & 1,703 & 95 & 214 & 309 & 652 & 0 & 60 \\
\hline $43 \mathrm{H}-3,45-47$ & 348.45 & 46 & 1,693 & 66 & 177 & 222 & 824 & 0 & 65 \\
\hline $43 \mathrm{H}-5,44-46$ & 351.44 & 65 & 1,368 & 115 & 233 & 267 & 504 & 0 & 56 \\
\hline $44 \mathrm{H}-1,44-46$ & 353.14 & 53 & 2,425 & 76 & 170 & 295 & 722 & 0 & 52 \\
\hline
\end{tabular}


Table T2 (continued). (Continued on next page.)

\begin{tabular}{|c|c|c|c|c|c|c|c|c|c|}
\hline \multirow{2}{*}{$\begin{array}{l}\text { Core, section, } \\
\text { interval }\end{array}$} & \multirow{2}{*}{$\begin{array}{l}\text { Depth } \\
\text { (mbsf) }\end{array}$} & \multicolumn{8}{|c|}{ Peak (cps) } \\
\hline & & Phyllosilicates & Quartz & K-feldspar & Plagioclase & Calcite & Dolomite & Ankerite & Halite \\
\hline $44 \mathrm{H}-3,44-46$ & 356.04 & 80 & 2,295 & 115 & 247 & 381 & 750 & 0 & 55 \\
\hline $45 X-1,44-46$ & 358.34 & 61 & 1,849 & 84 & 211 & 249 & 814 & 0 & 76 \\
\hline $45 X-3,45-47$ & 361.35 & 69 & 1,667 & 83 & 195 & 338 & 671 & 0 & 62 \\
\hline $46 X-1,45-47$ & 362.85 & 128 & 2,315 & 78 & 169 & 355 & 306 & 0 & 165 \\
\hline $46 X-3,43-45$ & 365.83 & 47 & 1,718 & 79 & 192 & 318 & 786 & 0 & 29 \\
\hline $47 \mathrm{H}-1,45-47$ & 368.45 & 74 & 2,436 & 154 & 223 & 325 & 685 & 0 & 66 \\
\hline $47 \mathrm{H}-3,42-44$ & 371.42 & 59 & 2,270 & 216 & 412 & 217 & 682 & 0 & 58 \\
\hline $48 \mathrm{H}-1,45-47$ & 373.65 & 71 & 2,232 & 218 & 226 & 386 & 996 & 0 & 140 \\
\hline $48 \mathrm{H}-3,45-47$ & 376.65 & 59 & 2,616 & 91 & 234 & 322 & 780 & 0 & 69 \\
\hline $48 \mathrm{H}-5,45-47$ & 379.65 & 72 & 3,175 & 472 & 328 & 365 & 915 & 0 & 28 \\
\hline $49 \mathrm{H}-1,44-46$ & 381.94 & 83 & 2,429 & 145 & 275 & 396 & 922 & 0 & 126 \\
\hline $49 \mathrm{H}-3,44-46$ & 384.94 & 35 & 1,513 & 86 & 172 & 170 & 754 & 0 & 24 \\
\hline $49 \mathrm{H}-4,44-46$ & 386.35 & 75 & 2,954 & 94 & 258 & 381 & 947 & 0 & 69 \\
\hline $50 \mathrm{H}-1,45-47$ & 388.35 & 136 & 2,122 & 179 & 144 & 448 & 145 & 0 & 162 \\
\hline $50 \mathrm{H}-3,45-47$ & 391.35 & 71 & 2,367 & 90 & 235 & 190 & 667 & 0 & 31 \\
\hline $50 \mathrm{H}-5,45-47$ & 393.85 & 70 & 1,705 & 57 & 192 & 238 & 433 & 0 & 28 \\
\hline $51 X-2,45-47$ & 396.45 & 52 & 2,253 & 156 & 320 & 299 & 635 & 0 & 57 \\
\hline $52 X-1,45-47$ & 397.25 & 75 & 3,820 & 212 & 257 & 248 & 829 & 0 & 58 \\
\hline $52 X-3,45-47$ & 400.25 & 67 & 2,621 & 79 & 207 & 279 & 723 & 0 & 56 \\
\hline $52 X-5,45-47$ & 403.25 & 71 & 2,165 & 166 & 244 & 315 & 581 & 0 & 58 \\
\hline $53 X-1,41-43$ & 406.81 & 58 & 3,877 & 309 & 255 & 239 & 576 & 0 & 52 \\
\hline $53 X-2,42-44$ & 408.32 & 73 & 5,192 & 86 & 146 & 193 & 145 & 0 & 137 \\
\hline $53 X-5,38-40$ & 412.78 & 56 & 3,121 & 247 & 246 & 296 & 614 & 0 & 56 \\
\hline $54 X-1,103-105$ & 417.03 & 43 & 4,303 & 253 & 319 & 254 & 612 & 0 & 44 \\
\hline $54 X-3,45-47$ & 419.45 & 48 & 3,646 & 224 & 572 & 157 & 404 & 0 & 56 \\
\hline $55 X-1,45-47$ & 426.15 & 77 & 2,606 & 146 & 284 & 381 & 567 & 0 & 68 \\
\hline $55 X-3,45-47$ & 429.15 & 59 & 2,155 & 122 & 353 & 370 & 567 & 0 & 53 \\
\hline $55 X-5,45-47$ & 432.15 & 50 & 2,278 & 116 & 211 & 267 & 417 & 0 & 36 \\
\hline $56 X-1,45-46$ & 435.75 & 66 & 2,217 & 111 & 277 & 326 & 587 & 0 & 61 \\
\hline $56 X-3,45-47$ & 438.75 & 55 & 1,456 & 142 & 228 & 209 & 485 & 0 & 98 \\
\hline $56 X-5,45-47$ & 441.75 & 82 & 2,226 & 92 & 192 & 130 & 239 & 0 & 35 \\
\hline $57 X-1,45-47$ & 445.45 & 64 & 4,103 & 283 & 242 & 116 & 302 & 0 & 70 \\
\hline $57 X-3,45-47$ & 448.45 & 57 & 1,568 & 405 & 349 & 124 & 254 & 0 & 43 \\
\hline $58 X-1,44-46$ & 455.04 & 69 & 2,847 & 71 & 202 & 91 & 394 & 0 & 47 \\
\hline $58 X-3,44-46$ & 458.04 & 88 & 4,089 & 212 & 381 & 153 & 474 & 0 & 81 \\
\hline $58 X-4,45-47$ & 459.55 & 80 & 2,153 & 164 & 231 & 160 & 303 & 0 & 31 \\
\hline $59 X-3,45-47$ & 466.59 & 49 & 1,823 & 104 & 174 & 409 & 669 & 0 & 52 \\
\hline $59 X-5,45-47$ & 469.59 & 87 & 3,431 & 273 & 217 & 332 & 997 & 0 & 47 \\
\hline $60 X-1,45-47$ & 474.35 & 71 & 3,639 & 159 & 378 & 427 & 924 & 0 & 172 \\
\hline $60 \times-3,45-47$ & 477.35 & 76 & 2,565 & 68 & 152 & 231 & 473 & 0 & 104 \\
\hline $60 X-5,45-47$ & 480.35 & 80 & 1,768 & 72 & 197 & 392 & 402 & 0 & 47 \\
\hline $61 X-1,45-47$ & 483.95 & 95 & 2,618 & 130 & 342 & 313 & 328 & 0 & 53 \\
\hline $61 X-3,45-47$ & 486.95 & 65 & 3,465 & 433 & 394 & 422 & 386 & 0 & 141 \\
\hline $61 X-5,47-49$ & 489.97 & 76 & 3,298 & 146 & 374 & 293 & 358 & 0 & 102 \\
\hline $62 X-1,51-53$ & 493.61 & 73 & 4,135 & 284 & 436 & 363 & 719 & 0 & 100 \\
\hline $62 X-3,45-47$ & 496.43 & 55 & 3,929 & 330 & 317 & 188 & 510 & 0 & 52 \\
\hline $62 X-5,45-47$ & 499.43 & 52 & 3,564 & 246 & 364 & 167 & 459 & 0 & 65 \\
\hline $63 X-1,50-52$ & 503.2 & 67 & 2,017 & 169 & 216 & 328 & 422 & 0 & 190 \\
\hline $63 X-3,47-49$ & 506.17 & 74 & 2,603 & 89 & 527 & 217 & 618 & 0 & 112 \\
\hline $63 X-5,43-45$ & 509.13 & 75 & 2,254 & 270 & 237 & 306 & 534 & 0 & 63 \\
\hline $64 X-1,45-47$ & 512.85 & 76 & 2,750 & 379 & 404 & 308 & 798 & 0 & 30 \\
\hline $64 X-3,45-47$ & 514.57 & 53 & 2,847 & 99 & 288 & 233 & 717 & 0 & 44 \\
\hline $65 X-1,45-47$ & 522.35 & 55 & 2,980 & 205 & 254 & 259 & 774 & 0 & 100 \\
\hline $65 X-3,45-47$ & 525.35 & 58 & 1,958 & 93 & 265 & 302 & 513 & 0 & 28 \\
\hline $65 X-5,45-47$ & 528.35 & 70 & 2,291 & 87 & 220 & 204 & 405 & 0 & 46 \\
\hline $66 X-1,44-46$ & 532.04 & 62 & 6,677 & 207 & 304 & 139 & 433 & 0 & 67 \\
\hline $67 X-1,45-47$ & 541.55 & 66 & 5,112 & 185 & 294 & 153 & 521 & 0 & 93 \\
\hline $67 X-3,45-47$ & 544.55 & 67 & 1,512 & 109 & 149 & 196 & 304 & 0 & 97 \\
\hline $67 X-5,45-47$ & 547.07 & 33 & 1,341 & 90 & 241 & 269 & 574 & 0 & 34 \\
\hline $68 X-1,46-49$ & 551.16 & 82 & 4,490 & 165 & 313 & 457 & 772 & 0 & 33 \\
\hline $68 X-3,45-47$ & 554.15 & 71 & 2,218 & 84 & 176 & 253 & 522 & 0 & 37 \\
\hline $68 X-5,45-47$ & 557.15 & 69 & 2,677 & 148 & 270 & 235 & 701 & 0 & 36 \\
\hline $69 X-1,45-47$ & 560.85 & 87 & 2,782 & 177 & 294 & 388 & 579 & 0 & 66 \\
\hline $69 X-3,45-47$ & 563.85 & 48 & 2,483 & 163 & 266 & 268 & 479 & 0 & 99 \\
\hline $70 X-1,44-46$ & 570.44 & 108 & 2,845 & 114 & 289 & 314 & 815 & 0 & 43 \\
\hline $70 X-3,44-46$ & 573.44 & 67 & 3,104 & 115 & 223 & 374 & 410 & 0 & 35 \\
\hline $70 X-5,44-46$ & 576.44 & 57 & 1,652 & 126 & 238 & 142 & 285 & 0 & 32 \\
\hline $71 X-1,46-48$ & 580.06 & 65 & 4,484 & 168 & 176 & 190 & 929 & 0 & 58 \\
\hline
\end{tabular}


Table T2 (continued).

\begin{tabular}{|c|c|c|c|c|c|c|c|c|c|}
\hline \multirow{2}{*}{$\begin{array}{l}\text { Core, section, } \\
\text { interval }\end{array}$} & \multirow{2}{*}{$\begin{array}{l}\text { Depth } \\
\text { (mbsf) }\end{array}$} & \multicolumn{8}{|c|}{ Peak (cps) } \\
\hline & & Phyllosilicates & Quartz & K-feldspar & Plagioclase & Calcite & Dolomite & Ankerite & Halite \\
\hline $71 X-1,47-49$ & 580.07 & 55 & 1,918 & 71 & 291 & 198 & 193 & 0 & 70 \\
\hline $71 X-3,44-46$ & 583.04 & 91 & 2,390 & 201 & 223 & 326 & 475 & 0 & 57 \\
\hline $71 X-3,45-47$ & 583.05 & 81 & 3,392 & 242 & 202 & 187 & 1069 & 0 & 545 \\
\hline $71 X-5,45-47$ & 586.05 & 82 & 2,304 & 68 & 264 & 252 & 258 & 0 & 44 \\
\hline $73 X-1,44-46$ & 593.64 & 84 & 3,997 & 440 & 637 & 242 & 803 & 0 & 55 \\
\hline $74 X-1,42-44$ & 599.02 & 82 & 1,905 & 111 & 260 & 301 & 475 & 0 & 95 \\
\hline $74 X-3,45-47$ & 602.05 & 59 & 1,507 & 109 & 233 & 296 & 418 & 0 & 86 \\
\hline $74 X-5,45-47$ & 605.05 & 34 & 2,952 & 26 & 226 & 38 & 256 & 0 & 71 \\
\hline
\end{tabular}

Note: $\mathrm{cps}=$ counts per second,$-=$ no data . 
Table T3. Bulk rock analyses quantified results. (Continued on next four pages.) (See table note.)

\begin{tabular}{|c|c|c|c|c|c|c|c|c|c|c|}
\hline \multirow{2}{*}{$\begin{array}{l}\text { Core, section, } \\
\text { interval }(\mathrm{cm})\end{array}$} & \multirow{2}{*}{$\begin{array}{l}\text { Depth } \\
\text { (mbsf) }\end{array}$} & \multicolumn{9}{|c|}{ Abundance (wt\%) } \\
\hline & & Phyllosilicates & Quartz & K-feldspar & Plagioclase & Calcite & Dolomite & Ankerite & Halite & Unquantified \\
\hline \multicolumn{11}{|l|}{ 308-U1320A- } \\
\hline $2 \mathrm{H}-1,44-46$ & 4.9 & 35.2 & 47.0 & 2.5 & 3.1 & 4.6 & 6.0 & 0.0 & 0.7 & 0.9 \\
\hline $2 \mathrm{H}-3,44-46$ & 7.9 & 19.3 & 42.8 & 13.8 & 11.2 & 1.7 & 4.3 & 0.0 & 0.0 & 6.9 \\
\hline $2 \mathrm{H}-5,44-46$ & 10.9 & 12.0 & 77.4 & 0.0 & 4.8 & 0.6 & 0.0 & 0.1 & 0.7 & 4.2 \\
\hline $3 \mathrm{H}-1,44-46$ & 14.4 & 21.6 & 57.3 & 4.2 & 1.8 & 2.4 & 2.3 & 0.0 & 2.8 & 7.6 \\
\hline $3 \mathrm{H}-3,60-62$ & 17.6 & 11.2 & 61.9 & 20.8 & 1.5 & 1.0 & 1.3 & 0.0 & 0.5 & 1.7 \\
\hline $3 \mathrm{H}-5,45-47$ & 20.5 & 31.2 & 50.1 & 5.6 & 2.6 & 5.5 & 3.0 & 0.0 & 0.8 & 1.2 \\
\hline $4 \mathrm{H}-1,44-46$ & 23.9 & 13.3 & 81.0 & 0.6 & 0.7 & 0.4 & 1.0 & 0.0 & 0.4 & 2.6 \\
\hline $4 \mathrm{H}-3,44-46$ & 26.9 & 47.3 & 32.9 & 2.8 & 3.7 & 5.8 & 3.8 & 0.0 & 1.4 & 2.2 \\
\hline $4 \mathrm{H}-5,44-46$ & 29.9 & 46.7 & 27.9 & 2.6 & 3.0 & 9.4 & 3.0 & 0.0 & 1.2 & 6.2 \\
\hline $5 \mathrm{H}-1,46-48$ & 33.5 & 41.6 & 25.7 & 3.6 & 4.7 & 7.8 & 5.1 & 0.0 & 1.8 & 9.9 \\
\hline $5 \mathrm{H}-3,45-47$ & 36.5 & 44.1 & 32.6 & 2.9 & 4.6 & 8.7 & 4.2 & 0.0 & 2.3 & 0.7 \\
\hline $5 \mathrm{H}-5,46-48$ & 39.5 & 22.4 & 53.2 & 4.9 & 5.3 & 4.9 & 6.1 & 0.0 & 0.7 & 2.4 \\
\hline $6 \mathrm{H}-1,45-47$ & 43.0 & 19.3 & 58.0 & 8.5 & 4.8 & 3.8 & 3.0 & 0.0 & 0.7 & 2.1 \\
\hline $6 \mathrm{H}-3,44-47$ & 45.9 & 9.2 & 82.9 & 1.5 & 0.9 & 3.0 & 0.6 & 0.0 & 0.7 & 1.3 \\
\hline $6 \mathrm{H}-5,45-47$ & 49.0 & 11.1 & 66.8 & 2.1 & 3.5 & 0.8 & 0.7 & 0.0 & 0.7 & 14.3 \\
\hline $7 \mathrm{H}-1,44-47$ & 52.4 & - & - & - & - & - & - & - & - & - \\
\hline $7 \mathrm{H}-3,44-47$ & 55.4 & 9.2 & 82.0 & 1.5 & 1.3 & 1.4 & 3.1 & 0.0 & 0.7 & 0.9 \\
\hline $7 \mathrm{H}-5,44-47$ & 58.4 & 31.0 & 50.3 & 4.8 & 3.1 & 5.2 & 2.7 & 0.0 & 0.0 & 2.7 \\
\hline $8 \mathrm{H}-1,45-47$ & 61.1 & 38.0 & 36.5 & 4.0 & 3.8 & 8.7 & 5.2 & 0.0 & 1.5 & 2.4 \\
\hline $8 \mathrm{H}-3,45-47$ & 64.1 & 37.1 & 47.5 & 2.7 & 5.0 & 4.4 & 1.1 & 0.0 & 0.3 & 2.0 \\
\hline $8 \mathrm{H}-5,45-47$ & 66.1 & 16.2 & 77.4 & 2.1 & 0.4 & 0.7 & 0.0 & 0.0 & 0.8 & 2.4 \\
\hline $9 \mathrm{H}-1,45-47$ & 67.8 & 8.7 & 77.3 & 8.2 & 1.1 & 0.7 & 1.4 & 0.0 & 0.6 & 2.1 \\
\hline $11 X-1,42-44$ & 78.7 & 38.4 & 25.7 & 3.8 & 3.5 & 9.6 & 5.4 & 0.0 & 1.3 & 12.2 \\
\hline $12 X-1,45-47$ & 88.4 & 42.8 & 24.3 & 3.2 & 3.9 & 10.7 & 3.7 & 0.0 & 2.0 & 9.5 \\
\hline $12 X-3,45-47$ & 91.4 & 27.9 & 39.0 & 3.9 & 7.8 & 6.0 & 4.9 & 0.0 & 1.4 & 9.2 \\
\hline $14 \mathrm{X}-1,47-49$ & 107.6 & 32.4 & 45.7 & 3.7 & 8.5 & 6.4 & 1.9 & 0.0 & 0.5 & 0.8 \\
\hline $14 X-3,46-49$ & 110.6 & 29.5 & 52.1 & 1.1 & 3.4 & 5.6 & 6.0 & 0.0 & 0.5 & 1.8 \\
\hline $15 X-1,44-46$ & 117.1 & 28.2 & 27.4 & 6.5 & 4.2 & 11.1 & 9.9 & 0.0 & 1.5 & 11.3 \\
\hline $15 X-3,44-46$ & 120.1 & 42.5 & 27.0 & 3.1 & 3.7 & 9.5 & 6.2 & 0.0 & 1.1 & 7.1 \\
\hline $15 X-5,44-46$ & 123.1 & 45.7 & 28.9 & 3.1 & 3.5 & 9.7 & 5.1 & 0.0 & 0.3 & 3.7 \\
\hline $16 \mathrm{X}-1,107-109$ & 127.4 & 37.0 & 32.2 & 3.0 & 3.9 & 13.4 & 5.3 & 0.0 & 3.0 & 2.2 \\
\hline $16 X-3,45-47$ & 129.8 & 41.4 & 18.5 & 3.8 & 5.8 & 17.5 & 4.2 & 0.0 & 2.9 & 5.8 \\
\hline $16 X-5,41-43$ & 132.7 & 50.9 & 16.0 & 4.3 & 6.1 & 13.9 & 3.0 & 0.0 & 1.3 & 4.5 \\
\hline $17 X-1,41-43$ & 136.3 & 48.1 & 32.2 & 2.1 & 3.4 & 8.2 & 2.8 & 0.0 & 0.4 & 2.7 \\
\hline $17 X-3,45-47$ & 138.7 & 45.0 & 26.5 & 3.2 & 4.4 & 12.5 & 6.6 & 0.0 & 0.9 & 0.8 \\
\hline $17 X-5,44-46$ & 141.6 & 38.2 & 13.1 & 27.0 & 4.4 & 11.8 & 2.4 & 0.0 & 0.9 & 2.2 \\
\hline $19 X-1,45-47$ & 155.7 & 45.4 & 26.8 & 4.7 & 5.5 & 2.0 & 3.4 & 0.0 & 0.6 & 11.7 \\
\hline $19 X-3,45-47$ & 158.7 & 44.8 & 27.0 & 3.2 & 3.6 & 2.7 & 2.7 & 0.0 & 0.7 & 15.5 \\
\hline $19 X-5,45-47$ & 161.7 & 52.5 & 23.2 & 3.5 & 4.9 & 4.1 & 0.0 & 0.0 & 0.0 & 11.8 \\
\hline $20 X-1,46-48$ & 165.3 & 51.7 & 33.9 & 2.7 & 2.9 & 2.3 & 4.9 & 0.0 & 0.5 & 1.3 \\
\hline $20 X-1,46-48$ & 165.3 & 41.9 & 24.7 & 3.0 & 5.1 & 6.2 & 4.7 & 0.0 & 1.1 & 13.3 \\
\hline $20 X-3,44-46$ & 168.2 & 30.5 & 45.7 & 3.0 & 8.6 & 1.7 & 6.8 & 0.0 & 0.3 & 3.4 \\
\hline $22 X-1,44-46$ & 184.5 & 46.9 & 24.5 & 3.2 & 3.8 & 8.4 & 10.3 & 0.0 & 0.9 & 1.9 \\
\hline $22 X-3,42-44$ & 187.5 & 51.9 & 23.5 & 1.9 & 4.8 & 6.0 & 8.3 & 0.0 & 1.1 & 2.4 \\
\hline $22 X-5,44-46$ & 190.5 & 36.0 & 23.8 & 3.6 & 5.1 & 10.8 & 14.4 & 0.0 & 1.0 & 5.5 \\
\hline $23 X-1,47-49$ & 194.3 & 46.9 & 26.0 & 3.1 & 3.8 & 7.2 & 9.2 & 0.0 & 2.1 & 1.8 \\
\hline $23 X-3,47-49$ & 197.3 & 33.8 & 27.0 & 3.2 & 4.1 & 9.0 & 11.7 & 0.0 & 0.5 & 10.8 \\
\hline $23 X-5,47-49$ & 200.3 & 27.5 & 33.7 & 2.6 & 3.5 & 8.0 & 20.0 & 0.0 & 0.8 & 4.0 \\
\hline $24 X-1,42-44$ & 203.8 & 38.4 & 21.6 & 3.1 & 4.5 & 13.8 & 17.3 & 0.0 & 0.7 & 0.6 \\
\hline $24 X-3,41-43$ & 206.8 & 38.8 & 25.1 & 3.4 & 4.5 & 13.1 & 14.3 & 0.0 & 0.8 & 0.0 \\
\hline $24 X-5,41-43$ & 209.8 & 29.6 & 18.6 & 3.4 & 4.7 & 14.0 & 18.1 & 0.0 & 1.5 & 10.2 \\
\hline $25 X-1,44-47$ & 213.4 & 34.6 & 22.3 & 2.1 & 4.8 & 11.8 & 20.5 & 0.0 & 0.8 & 3.1 \\
\hline $25 X-3,47-48$ & 216.5 & 27.6 & 17.9 & 3.6 & 5.7 & 14.0 & 15.3 & 0.0 & 1.2 & 14.8 \\
\hline $25 X-5,41-43$ & 219.4 & 36.0 & 23.9 & 3.0 & 7.4 & 14.5 & 13.0 & 0.0 & 1.2 & 1.0 \\
\hline $26 X-1,45-47$ & 223.1 & 27.9 & 25.0 & 4.4 & 5.2 & 11.5 & 23.2 & 0.0 & 1.0 & 1.7 \\
\hline $26 X-3,46-48$ & 225.9 & 33.5 & 19.7 & 5.0 & 6.6 & 12.5 & 13.9 & 0.0 & 0.7 & 8.2 \\
\hline $26 X-5,44-46$ & 228.9 & 29.9 & 20.9 & 3.2 & 4.6 & 12.3 & 15.1 & 0.0 & 1.4 & 12.6 \\
\hline $27 X-1,44-46$ & 232.6 & 35.6 & 20.4 & 4.6 & 6.7 & 8.6 & 11.1 & 0.0 & 1.3 & 11.8 \\
\hline $27 X-3,44-46$ & 235.6 & 22.3 & 17.9 & 2.0 & 5.5 & 12.4 & 11.8 & 0.0 & 1.1 & 27.0 \\
\hline $27 X-5,44-46$ & 238.6 & 40.1 & 20.0 & 2.8 & 5.3 & 13.4 & 14.2 & 0.0 & 0.8 & 3.4 \\
\hline $28 X-1,45-47$ & 242.4 & 30.7 & 23.8 & 2.1 & 5.6 & 14.5 & 15.3 & 0.0 & 0.7 & 7.4 \\
\hline $28 X-3,45-47$ & 245.4 & 27.8 & 21.9 & 4.5 & 5.3 & 12.3 & 17.3 & 0.0 & 0.8 & 10.0 \\
\hline $28 X-5,45-47$ & 248.4 & 28.7 & 29.8 & 2.5 & 4.7 & 13.0 & 12.7 & 0.0 & 0.9 & 7.8 \\
\hline $29 X-1,42-44$ & 251.9 & 37.7 & 18.8 & 3.5 & 7.6 & 13.9 & 15.5 & 0.0 & 0.6 & 2.4 \\
\hline $29 X-3,42-44$ & 254.9 & 24.9 & 22.5 & 4.2 & 5.0 & 12.8 & 17.9 & 0.0 & 0.7 & 12.1 \\
\hline $29 X-5,42-44$ & 257.9 & 26.4 & 26.1 & 4.0 & 6.0 & 15.4 & 19.6 & 0.0 & 0.7 & 1.9 \\
\hline $30 X-1,44-46$ & 261.5 & 30.8 & 23.6 & 3.3 & 7.3 & 13.1 & 18.7 & 0.0 & 0.7 & 2.6 \\
\hline $30 X-3,44-46$ & 264.5 & 34.0 & 26.3 & 3.2 & 10.0 & 13.8 & 10.9 & 0.0 & 0.6 & 1.2 \\
\hline
\end{tabular}


Table T3 (continued). (Continued on next page.)

\begin{tabular}{|c|c|c|c|c|c|c|c|c|c|c|}
\hline \multirow{2}{*}{$\begin{array}{l}\text { Core, section, } \\
\text { interval }(\mathrm{cm})\end{array}$} & \multirow{2}{*}{$\begin{array}{l}\text { Depth } \\
\text { (mbsf) }\end{array}$} & \multicolumn{9}{|c|}{ Abundance (wt\%) } \\
\hline & & Phyllosilicates & Quartz & K-feldspar & Plagioclase & Calcite & Dolomite & Ankerite & Halite & Unquantified \\
\hline $30 X-5,43-45$ & 267.5 & 40.8 & 22.7 & 3.0 & 5.9 & 11.9 & 9.9 & 0.0 & 0.5 & 5.2 \\
\hline $31 X-1,45-47$ & 271.2 & 27.5 & 20.5 & 4.3 & 7.1 & 13.6 & 11.0 & 0.0 & 0.6 & 15.4 \\
\hline $31 X-3,45-47$ & 274.2 & 28.7 & 21.6 & 2.2 & 8.7 & 14.6 & 9.9 & 0.0 & 0.9 & 13.6 \\
\hline $31 X-5,45-47$ & 277.2 & 29.5 & 20.6 & 4.5 & 9.7 & 12.3 & 9.8 & 0.0 & 1.0 & 12.7 \\
\hline $32 X-1,44-46$ & 280.7 & 28.8 & 26.4 & 5.5 & 6.0 & 12.4 & 10.0 & 0.0 & 1.0 & 9.8 \\
\hline $32 X-3,42-44$ & 283.7 & 30.8 & 23.9 & 3.1 & 5.8 & 16.5 & 12.0 & 0.0 & 2.2 & 5.8 \\
\hline $32 X-5,43-45$ & 286.7 & 39.5 & 19.1 & 2.7 & 7.3 & 13.2 & 14.6 & 0.0 & 0.8 & 2.9 \\
\hline $33 X-1,44-46$ & 290.3 & 38.0 & 22.1 & 7.0 & 6.4 & 13.0 & 11.4 & 0.0 & 0.9 & 1.1 \\
\hline $33 X-3,44-46$ & 293.3 & 31.3 & 27.7 & 3.5 & 5.9 & 15.0 & 13.1 & 0.0 & 1.3 & 2.3 \\
\hline $33 X-5,45-47$ & 296.4 & 26.0 & 21.7 & 2.6 & 5.4 & 15.0 & 12.9 & 0.0 & 1.4 & 15.1 \\
\hline \multicolumn{11}{|l|}{ 308-U1324B- } \\
\hline $1 \mathrm{H}-1,45-47$ & 0.5 & 47.1 & 21.8 & 4.8 & 4.3 & 7.5 & 7.7 & 0.0 & 5.2 & 1.7 \\
\hline $1 \mathrm{H}-3,45-47$ & 3.5 & 56.0 & 21.4 & 2.5 & 4.0 & 3.3 & 4.7 & 0.0 & 3.4 & 4.7 \\
\hline $2 \mathrm{H}-1,45-47$ & 4.3 & 39.7 & 27.9 & 2.9 & 5.0 & 4.8 & 5.0 & 0.0 & 3.2 & 11.6 \\
\hline $2 \mathrm{H}-3,45-47$ & 7.3 & 43.6 & 27.7 & 2.9 & 7.0 & 5.1 & 9.9 & 0.0 & 2.8 & 1.1 \\
\hline $2 \mathrm{H}-5,34-36$ & 10.1 & 37.0 & 22.7 & 3.1 & 3.6 & 14.9 & 8.9 & 0.0 & 1.5 & 8.4 \\
\hline $3 \mathrm{H}-1,44-46$ & 13.7 & 41.7 & 25.7 & 2.8 & 4.0 & 7.9 & 10.3 & 0.0 & 2.4 & 5.2 \\
\hline $3 \mathrm{H}-3,44-46$ & 16.7 & 37.7 & 24.6 & 2.6 & 3.5 & 10.5 & 7.7 & 0.0 & 1.8 & 11.7 \\
\hline $3 \mathrm{H}-5,45-47$ & 19.8 & 29.0 & 35.1 & 2.1 & 7.0 & 9.9 & 12.5 & 0.0 & 3.0 & 1.5 \\
\hline $4 \mathrm{H}-1,44-47$ & 23.2 & 44.9 & 26.5 & 2.8 & 3.8 & 7.0 & 10.7 & 0.0 & 1.2 & 3.2 \\
\hline $4 \mathrm{H}-3,44-47$ & 26.2 & 42.8 & 27.1 & 3.5 & 4.6 & 9.3 & 8.3 & 0.0 & 1.4 & 3.0 \\
\hline $4 \mathrm{H}-5,45-47$ & 29.3 & 43.2 & 27.1 & 3.5 & 4.6 & 9.3 & 8.3 & 0.0 & 1.4 & 2.6 \\
\hline $5 \mathrm{H}-1,45-47$ & 32.8 & 47.2 & 22.0 & 2.9 & 4.5 & 9.3 & 10.7 & 0.0 & 1.8 & 1.6 \\
\hline $5 \mathrm{H}-3,45-47$ & 35.8 & 42.0 & 26.0 & 2.9 & 4.5 & 10.8 & 10.9 & 0.0 & 1.5 & 1.4 \\
\hline $5 \mathrm{H}-5,45-47$ & 38.8 & 34.9 & 32.9 & 2.1 & 5.9 & 8.0 & 8.8 & 0.0 & 4.9 & 2.5 \\
\hline $6 \mathrm{H}-1,45-47$ & 42.3 & 43.1 & 19.2 & 3.6 & 4.1 & 12.3 & 6.9 & 0.0 & 1.9 & 9.0 \\
\hline $6 \mathrm{H}-3,45-47$ & 45.3 & 41.3 & 22.3 & 3.8 & 7.5 & 16.0 & 6.2 & 0.0 & 1.7 & 1.2 \\
\hline $6 \mathrm{H}-5,45-47$ & 48.3 & 40.7 & 24.5 & 3.1 & 3.1 & 14.1 & 10.1 & 0.0 & 1.9 & 2.5 \\
\hline $7 \mathrm{H}-1,45-47$ & 51.8 & 48.1 & 21.7 & 3.1 & 4.2 & 9.9 & 8.3 & 0.0 & 1.5 & 3.3 \\
\hline $7 \mathrm{H}-3,45-47$ & 54.8 & 39.0 & 30.8 & 2.1 & 9.6 & 8.5 & 8.1 & 0.0 & 1.4 & 0.6 \\
\hline $7 \mathrm{H}-5,45-47$ & 57.8 & 45.3 & 25.3 & 2.6 & 4.2 & 8.8 & 9.6 & 0.0 & 1.3 & 2.9 \\
\hline $8 \mathrm{H}-1,44-47$ & 61.2 & 42.3 & 24.1 & 2.3 & 4.0 & 13.3 & 11.2 & 0.0 & 1.3 & 1.5 \\
\hline $8 \mathrm{H}-3,45-47$ & 64.3 & 37.8 & 18.7 & 3.6 & 5.1 & 18.0 & 6.0 & 0.0 & 1.0 & 9.9 \\
\hline $8 \mathrm{H}-5,45-47$ & 67.3 & 40.4 & 23.9 & 3.3 & 3.7 & 9.6 & 11.4 & 0.0 & 1.0 & 6.8 \\
\hline $9 \mathrm{H}-1,44-46$ & 70.7 & 39.8 & 26.5 & 5.3 & 2.7 & 8.7 & 10.2 & 0.0 & 5.9 & 1.0 \\
\hline $9 \mathrm{H}-3,45-47$ & 73.8 & 46.6 & 22.7 & 2.5 & 4.0 & 8.4 & 15.0 & 0.0 & 0.5 & 0.4 \\
\hline $9 \mathrm{H}-5,45-47$ & 76.8 & 37.8 & 26.9 & 2.1 & 5.0 & 8.5 & 10.5 & 0.0 & 2.0 & 7.4 \\
\hline $10 \mathrm{H}-1,43-45$ & 80.2 & 41.5 & 29.1 & 2.3 & 4.0 & 8.0 & 11.7 & 0.0 & 2.2 & 1.2 \\
\hline $10 \mathrm{H}-3,43-45$ & 83.2 & 36.8 & 22.0 & 3.4 & 4.7 & 7.7 & 12.8 & 0.0 & 1.3 & 11.4 \\
\hline $10 \mathrm{H}-5,43-45$ & 86.2 & 44.1 & 31.7 & 3.5 & 3.7 & 5.6 & 9.7 & 0.0 & 0.8 & 1.0 \\
\hline $11 \mathrm{H}-1,39-41$ & 89.7 & 37.0 & 27.8 & 5.2 & 5.4 & 9.5 & 12.0 & 0.0 & 1.7 & 1.5 \\
\hline $11 \mathrm{H}-3,44-46$ & 92.7 & 44.7 & 25.4 & 3.8 & 5.9 & 6.1 & 9.6 & 0.0 & 0.6 & 3.9 \\
\hline $11 \mathrm{H}-5,45-47$ & 95.8 & 39.2 & 23.9 & 3.8 & 5.9 & 7.4 & 10.4 & 0.0 & 1.3 & 8.0 \\
\hline $12 \mathrm{H}-1,45-47$ & 99.3 & 37.5 & 18.2 & 2.6 & 4.7 & 20.8 & 12.2 & 0.0 & 1.4 & 2.7 \\
\hline $12 \mathrm{H}-3,45-47$ & 102.3 & 43.2 & 24.0 & 3.1 & 5.1 & 10.6 & 11.5 & 0.0 & 0.9 & 1.6 \\
\hline $12 \mathrm{H}-5,45-47$ & 105.3 & 42.3 & 26.8 & 3.1 & 5.1 & 9.6 & 12.2 & 0.0 & 0.7 & 0.3 \\
\hline $13 \mathrm{H}-1,40-42$ & 108.7 & 36.6 & 36.3 & 1.5 & 5.5 & 5.6 & 13.2 & 0.0 & 0.8 & 0.6 \\
\hline $13 \mathrm{H}-3,45-47$ & 111.8 & 31.1 & 25.5 & 4.3 & 4.1 & 9.2 & 15.3 & 0.0 & 0.9 & 9.6 \\
\hline $13 \mathrm{H}-5,44-46$ & 114.7 & 37.0 & 31.7 & 3.2 & 4.5 & 10.5 & 12.3 & 0.0 & 0.3 & 0.4 \\
\hline $14 \mathrm{H}-1,44-46$ & 118.2 & 44.8 & 22.6 & 2.7 & 5.0 & 8.9 & 11.0 & 0.0 & 0.6 & 4.5 \\
\hline $14 \mathrm{H}-2,45-47$ & 119.2 & 29.6 & 30.6 & 2.4 & 5.3 & 9.0 & 13.7 & 0.0 & 0.9 & 8.5 \\
\hline $14 \mathrm{H}-3,45-47$ & 120.1 & 33.6 & 29.5 & 2.1 & 4.5 & 11.4 & 17.5 & 0.0 & 0.8 & 0.6 \\
\hline $14 \mathrm{H}-5,45-47$ & 122.1 & 35.4 & 30.5 & 3.6 & 5.6 & 7.1 & 16.0 & 0.0 & 1.1 & 0.6 \\
\hline $15 \mathrm{H}-1,45-47$ & 127.8 & - & - & - & - & - & - & - & - & - \\
\hline $15 \mathrm{H}-3,45-47$ & 130.8 & 36.1 & 24.1 & 4.0 & 4.1 & 12.1 & 17.8 & 0.0 & 1.1 & 0.8 \\
\hline $15 \mathrm{H}-5,45-47$ & 133.8 & 33.9 & 24.9 & 3.0 & 4.5 & 12.8 & 18.8 & 0.0 & 0.8 & 1.2 \\
\hline $16 \mathrm{H}-1,43-45$ & 136.7 & 25.8 & 22.5 & 3.6 & 6.2 & 13.6 & 18.8 & 0.0 & 1.9 & 7.8 \\
\hline $16 \mathrm{H}-3,43-45$ & 139.2 & 25.5 & 34.1 & 3.1 & 6.4 & 8.1 & 22.0 & 0.0 & 0.6 & 0.2 \\
\hline $16 \mathrm{H}-5,45-47$ & 141.7 & - & - & - & - & - & - & - & - & - \\
\hline $17 \mathrm{H}-1,45-47$ & 145.4 & 33.0 & 21.6 & 2.9 & 5.3 & 14.9 & 19.2 & 0.0 & 1.2 & 1.9 \\
\hline $17 \mathrm{H}-3,45-47$ & 148.4 & 31.6 & 22.6 & 3.5 & 4.8 & 14.0 & 19.9 & 0.0 & 2.4 & 1.3 \\
\hline $17 \mathrm{H}-5,45-47$ & 151.4 & 27.7 & 24.8 & 3.0 & 5.9 & 14.0 & 20.1 & 0.0 & 0.8 & 3.7 \\
\hline $18 \mathrm{H}-1,44-46$ & 154.1 & 40.8 & 34.8 & 4.3 & 4.9 & 2.9 & 4.0 & 0.0 & 1.5 & 6.9 \\
\hline $18 \mathrm{H}-2,46-48$ & 155.7 & 26.1 & 21.3 & 3.8 & 5.2 & 13.2 & 22.7 & 0.0 & 0.7 & 6.9 \\
\hline $18 \mathrm{H}-3,49-51$ & 157.2 & 22.4 & 25.9 & 3.1 & 6.2 & 12.8 & 24.4 & 0.0 & 1.4 & 3.9 \\
\hline $19 \mathrm{H}-1,44-46$ & 162.4 & 26.9 & 19.2 & 6.0 & 7.1 & 17.1 & 15.0 & 0.0 & 0.5 & 8.2 \\
\hline $19 \mathrm{H}-3,44-46$ & 165.4 & 42.6 & 21.0 & 5.3 & 5.9 & 8.6 & 9.7 & 0.0 & 1.3 & 5.8 \\
\hline $19 \mathrm{H}-5,44-46$ & 168.4 & 36.5 & 24.2 & 2.8 & 5.7 & 12.9 & 16.1 & 0.0 & 1.2 & 0.7 \\
\hline
\end{tabular}


Table T3 (continued). (Continued on next page.)

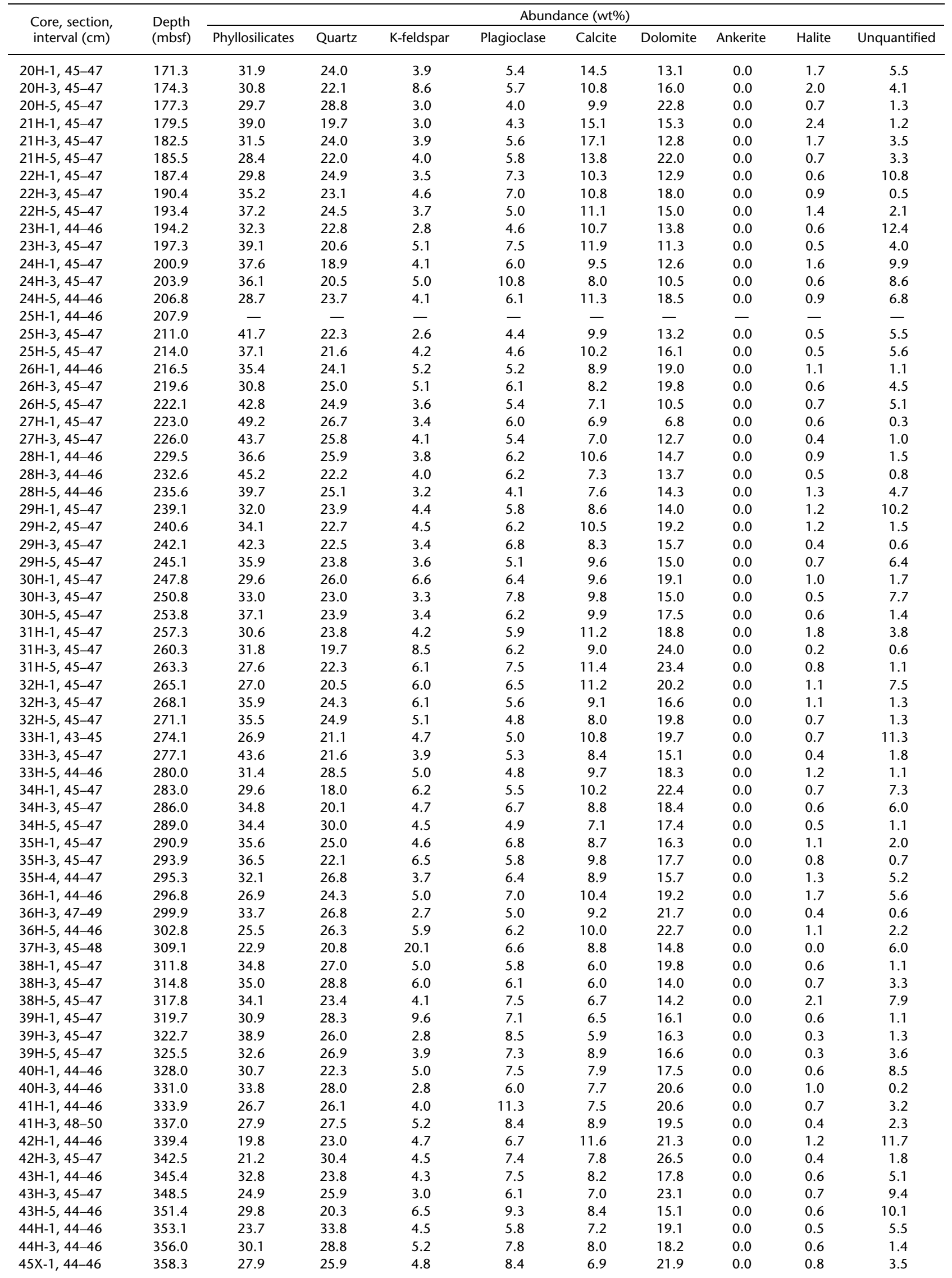


Table T3 (continued). (Continued on next page.)

\begin{tabular}{|c|c|c|c|c|c|c|c|c|c|c|}
\hline \multirow{2}{*}{$\begin{array}{l}\text { Core, section, } \\
\text { interval }(\mathrm{cm})\end{array}$} & \multirow{2}{*}{$\begin{array}{l}\text { Depth } \\
\text { (mbsf) }\end{array}$} & \multicolumn{9}{|c|}{ Abundance (wt\%) } \\
\hline & & Phyllosilicates & Quartz & K-feldspar & Plagioclase & Calcite & Dolomite & Ankerite & Halite & Unquantified \\
\hline $45 X-3,45-47$ & 361.4 & 30.7 & 23.6 & 3.8 & 6.9 & 8.9 & 19.2 & 0.0 & 0.7 & 6.4 \\
\hline $46 X-1,45-47$ & 362.9 & 45.0 & 29.3 & 3.5 & 5.9 & 7.2 & 6.9 & 0.0 & 1.2 & 0.9 \\
\hline $46 X-3,43-45$ & 365.8 & 24.1 & 24.6 & 3.6 & 6.7 & 9.5 & 22.2 & 0.0 & 1.9 & 7.4 \\
\hline $47 \mathrm{H}-1,45-47$ & 368.5 & 29.9 & 31.3 & 6.3 & 7.3 & 7.1 & 17.2 & 0.0 & 0.7 & 0.2 \\
\hline $47 \mathrm{H}-3,42-44$ & 371.4 & 23.9 & 29.0 & 9.3 & 13.8 & 4.8 & 16.8 & 0.0 & 0.6 & 1.8 \\
\hline $48 \mathrm{H}-1,45-47$ & 373.7 & 27.4 & 27.0 & 8.3 & 6.1 & 7.0 & 21.9 & 0.0 & 1.5 & 0.8 \\
\hline $48 \mathrm{H}-3,45-47$ & 376.7 & 24.4 & 34.1 & 4.0 & 8.0 & 7.3 & 19.6 & 0.0 & 0.7 & 1.8 \\
\hline $48 \mathrm{H}-5,45-47$ & 379.7 & 20.9 & 36.0 & 10.6 & 8.1 & 5.3 & 18.1 & 0.0 & 0.3 & 0.8 \\
\hline $49 \mathrm{H}-1,44-46$ & 381.9 & 31.1 & 26.3 & 5.6 & 8.2 & 7.1 & 21.3 & 0.0 & 0.3 & 0.0 \\
\hline $49 \mathrm{H}-3,44-46$ & 384.9 & 20.7 & 23.6 & 4.8 & 7.0 & 10.9 & 24.9 & 0.0 & 0.2 & 7.9 \\
\hline $49 \mathrm{H}-4,44-46$ & 386.4 & 27.5 & 32.9 & 4.1 & 8.0 & 5.7 & 20.8 & 0.0 & 0.7 & 0.3 \\
\hline $50 \mathrm{H}-1,45-47$ & 388.4 & 43.8 & 26.3 & 8.0 & 5.1 & 10.6 & 3.8 & 0.0 & 1.7 & 0.6 \\
\hline $50 \mathrm{H}-3,45-47$ & 391.4 & 28.9 & 31.5 & 4.9 & 8.9 & 5.3 & 17.5 & 0.0 & 0.3 & 2.8 \\
\hline $50 \mathrm{H}-5,45-47$ & 393.9 & 34.8 & 25.9 & 3.6 & 7.6 & 7.5 & 13.0 & 0.0 & 0.3 & 7.4 \\
\hline $51 X-2,45-47$ & 396.5 & 22.5 & 30.4 & 6.9 & 10.9 & 7.6 & 17.0 & 0.0 & 0.6 & 4.1 \\
\hline $52 X-1,45-47$ & 397.3 & 21.7 & 40.2 & 7.4 & 7.0 & 4.7 & 17.3 & 0.0 & 0.6 & 1.0 \\
\hline $52 X-3,45-47$ & 400.3 & 27.6 & 34.0 & 3.5 & 7.0 & 6.3 & 18.0 & 0.0 & 0.6 & 3.1 \\
\hline $52 X-5,45-47$ & 403.3 & 29.7 & 28.5 & 7.4 & 8.4 & 7.2 & 14.7 & 0.0 & 0.6 & 3.4 \\
\hline $53 X-1,41-43$ & 406.8 & 21.7 & 41.3 & 12.1 & 7.5 & 4.1 & 12.3 & 0.0 & 0.5 & 0.6 \\
\hline $53 \mathrm{X}-2,42-44$ & 408.3 & 27.9 & 54.2 & 3.5 & 4.6 & 4.0 & 3.4 & 0.0 & 1.3 & 1.1 \\
\hline $53 X-5,38-40$ & 412.8 & 22.8 & 35.1 & 10.7 & 8.3 & 6.6 & 15.2 & 0.0 & 0.6 & 0.7 \\
\hline $54 \mathrm{X}-1,103-105$ & 417.0 & 16.0 & 46.9 & 9.5 & 9.2 & 4.3 & 13.5 & 0.0 & 0.4 & 0.2 \\
\hline $54 X-3,45-47$ & 419.5 & 18.2 & 41.5 & 9.1 & 17.9 & 3.3 & 9.3 & 0.0 & 0.5 & 0.2 \\
\hline $55 X-1,45-47$ & 426.2 & 30.1 & 32.6 & 5.5 & 8.7 & 7.7 & 13.6 & 0.0 & 0.7 & 1.3 \\
\hline $55 X-3,45-47$ & 429.2 & 25.6 & 29.3 & 5.4 & 12.1 & 9.5 & 15.3 & 0.0 & 0.5 & 2.2 \\
\hline $55 X-5,45-47$ & 432.2 & 24.7 & 33.7 & 6.1 & 8.2 & 8.0 & 12.5 & 0.0 & 1.5 & 5.4 \\
\hline $56 \mathrm{X}-1,45-46$ & 435.8 & 28.6 & 30.1 & 4.9 & 9.5 & 8.3 & 15.9 & 0.0 & 0.6 & 2.1 \\
\hline $56 \mathrm{X}-3,45-47$ & 438.8 & 27.2 & 24.8 & 7.4 & 9.0 & 6.9 & 14.2 & 0.0 & 1.9 & 8.6 \\
\hline $56 X-5,45-47$ & 441.8 & 35.3 & 33.0 & 4.9 & 7.5 & 4.3 & 7.9 & 0.0 & 1.3 & 5.8 \\
\hline $57 X-1,45-47$ & 445.5 & 24.7 & 46.9 & 10.2 & 7.2 & 2.4 & 6.1 & 0.0 & 0.7 & 1.8 \\
\hline $57 X-3,45-47$ & 448.5 & 28.0 & 26.8 & 18.9 & 12.9 & 2.8 & 6.3 & 0.0 & 0.4 & 3.9 \\
\hline $58 \mathrm{X}-1,44-46$ & 455.0 & 32.6 & 37.6 & 4.0 & 6.6 & 3.0 & 10.5 & 0.0 & 0.5 & 5.3 \\
\hline $58 X-3,44-46$ & 458.0 & 30.5 & 41.1 & 6.8 & 10.0 & 2.4 & 8.2 & 0.0 & 0.8 & 0.2 \\
\hline $58 X-4,45-47$ & 459.6 & 33.6 & 28.5 & 8.1 & 8.8 & 5.6 & 10.6 & 0.0 & 0.3 & 4.7 \\
\hline $59 X-3,45-47$ & 466.6 & 25.8 & 26.6 & 6.3 & 6.7 & 11.0 & 19.0 & 0.0 & 0.5 & 4.2 \\
\hline $59 X-5,45-47$ & 469.6 & 28.1 & 32.2 & 8.3 & 5.8 & 4.9 & 19.9 & 0.0 & 0.4 & 0.4 \\
\hline $60 X-1,45-47$ & 474.4 & 22.4 & 35.5 & 6.1 & 8.9 & 6.8 & 18.6 & 0.0 & 1.6 & 0.0 \\
\hline $60 X-3,45-47$ & 477.4 & 30.9 & 32.7 & 3.8 & 5.8 & 7.8 & 13.9 & 0.0 & 1.0 & 4.2 \\
\hline $60 \times-5,45-47$ & 480.4 & 34.6 & 25.7 & 4.0 & 7.3 & 10.5 & 11.8 & 0.0 & 0.5 & 5.6 \\
\hline $61 X-1,45-47$ & 484.0 & 35.8 & 31.0 & 5.2 & 10.6 & 6.4 & 7.5 & 0.0 & 0.5 & 2.9 \\
\hline $61 X-3,45-47$ & 487.0 & 23.6 & 37.9 & 11.6 & 10.3 & 7.8 & 7.9 & 0.0 & 0.4 & 0.7 \\
\hline $61 X-5,47-49$ & 490.0 & 28.1 & 38.4 & 5.8 & 11.4 & 5.9 & 8.0 & 0.0 & 0.9 & 1.5 \\
\hline $62 X-1,51-53$ & 493.6 & 22.3 & 40.0 & 9.1 & 10.5 & 5.6 & 12.0 & 0.0 & 0.1 & 0.4 \\
\hline $62 X-3,45-47$ & 496.4 & 19.9 & 43.7 & 11.6 & 9.1 & 3.7 & 10.7 & 0.0 & 0.5 & 0.9 \\
\hline $62 X-5,45-47$ & 499.4 & 19.9 & 41.6 & 9.5 & 11.4 & 4.6 & 11.3 & 0.0 & 0.6 & 1.2 \\
\hline $63 X-1,50-52$ & 503.2 & 29.3 & 27.9 & 7.1 & 7.0 & 9.0 & 12.0 & 0.0 & 1.8 & 5.8 \\
\hline $63 X-3,47-49$ & 506.2 & 27.5 & 31.1 & 3.5 & 16.0 & 4.4 & 13.8 & 0.0 & 1.0 & 2.8 \\
\hline $63 X-5,43-45$ & 509.1 & 29.0 & 28.5 & 11.0 & 7.2 & 7.5 & 13.7 & 0.0 & 0.6 & 2.6 \\
\hline $64 \mathrm{X}-1,45-47$ & 512.9 & 24.4 & 29.1 & 13.6 & 11.3 & 5.1 & 15.8 & 0.0 & 0.3 & 0.5 \\
\hline $64 X-3,45-47$ & 514.6 & 21.8 & 35.3 & 3.9 & 8.9 & 5.8 & 18.3 & 0.0 & 1.0 & 5.0 \\
\hline $65 X-1,45-47$ & 522.4 & 20.7 & 35.4 & 8.2 & 7.9 & 5.3 & 17.7 & 0.0 & 0.9 & 3.8 \\
\hline $65 X-3,45-47$ & 525.4 & 27.9 & 27.6 & 5.7 & 9.3 & 8.6 & 14.0 & 0.0 & 0.3 & 6.7 \\
\hline $65 X-5,45-47$ & 528.4 & 28.8 & 29.9 & 4.8 & 7.6 & 7.5 & 12.6 & 0.0 & 0.4 & 8.5 \\
\hline $66 \mathrm{X}-1,44-46$ & 532.0 & 18.8 & 53.7 & 7.3 & 8.0 & 2.5 & 8.4 & 0.0 & 0.6 & 0.6 \\
\hline $67 X-1,45-47$ & 541.6 & 21.7 & 50.3 & 7.5 & 8.2 & 1.6 & 9.1 & 0.0 & 0.8 & 0.8 \\
\hline $67 X-3,45-47$ & 544.6 & 33.4 & 32.7 & 5.4 & 5.7 & 6.3 & 9.3 & 0.0 & 0.9 & 6.3 \\
\hline $67 X-5,45-47$ & 547.1 & 19.9 & 28.6 & 4.6 & 8.7 & 7.6 & 16.7 & 0.0 & 0.3 & 13.7 \\
\hline $68 \mathrm{X}-1,46-49$ & 551.2 & 26.8 & 35.8 & 5.6 & 8.7 & 7.3 & 15.4 & 0.0 & 0.3 & 0.1 \\
\hline $68 X-3,45-47$ & 554.2 & 32.6 & 29.7 & 3.4 & 5.6 & 6.5 & 13.1 & 0.0 & 0.3 & 8.9 \\
\hline $68 X-5,45-47$ & 557.2 & 26.8 & 32.7 & 5.9 & 8.4 & 5.8 & 16.1 & 0.0 & 0.3 & 4.1 \\
\hline $69 X-1,45-47$ & 560.9 & 32.4 & 31.9 & 6.6 & 8.3 & 8.0 & 11.8 & 0.0 & 0.6 & 0.3 \\
\hline $69 X-3,45-47$ & 563.9 & 24.8 & 32.1 & 7.6 & 9.0 & 6.8 & 12.9 & 0.0 & 0.9 & 6.0 \\
\hline $70 \mathrm{X}-1,44-46$ & 570.4 & 34.9 & 30.9 & 3.1 & 7.7 & 5.1 & 17.5 & 0.0 & 0.2 & 0.7 \\
\hline $70 X-3,44-46$ & 573.4 & 26.5 & 38.0 & 5.7 & 7.0 & 8.9 & 10.6 & 0.0 & 0.3 & 3.1 \\
\hline $70 X-5,44-46$ & 576.4 & 31.4 & 27.9 & 8.6 & 11.0 & 5.1 & 9.8 & 0.0 & 0.3 & 6.0 \\
\hline $71 X-1,46-48$ & 580.1 & 21.8 & 44.6 & 6.5 & 5.2 & 2.0 & 18.5 & 0.0 & 0.5 & 0.9 \\
\hline $71 X-1,47-49$ & 580.1 & 25.9 & 30.6 & 4.7 & 11.1 & 8.1 & 8.7 & 0.0 & 0.7 & 10.4 \\
\hline $71 X-3,44-46$ & 583.0 & 35.0 & 29.0 & 8.2 & 7.1 & 6.9 & 11.1 & 0.0 & 0.5 & 2.2 \\
\hline
\end{tabular}


Table T3 (continued).

\begin{tabular}{|c|c|c|c|c|c|c|c|c|c|c|}
\hline \multirow{2}{*}{$\begin{array}{l}\text { Core, section, } \\
\text { interval }(\mathrm{cm})\end{array}$} & \multirow{2}{*}{$\begin{array}{l}\text { Depth } \\
\text { (mbsf) }\end{array}$} & \multicolumn{9}{|c|}{ Abundance (wt\%) } \\
\hline & & Phyllosilicates & Quartz & K-feldspar & Plagioclase & Calcite & Dolomite & Ankerite & Halite & Unquantified \\
\hline $71 X-3,45-47$ & 583.1 & 23.7 & 33.7 & 9.0 & 5.4 & 3.0 & 19.7 & 0.0 & 5.2 & 0.5 \\
\hline $73 X-1,44-46$ & 593.6 & 23.0 & 35.8 & 11.7 & 10.6 & 3.0 & 14.3 & 0.0 & 0.5 & 1.1 \\
\hline $74 X-1,42-44$ & 599.0 & 33.9 & 25.9 & 5.7 & 8.3 & 8.6 & 13.7 & 0.0 & 0.9 & 3.2 \\
\hline $74 X-3,45-47$ & 602.1 & 28.7 & 29.7 & 5.5 & 7.9 & 8.0 & 11.0 & 0.0 & 1.0 & 8.3 \\
\hline $74 X-5,45-47$ & 605.1 & 22.8 & 41.5 & 3.5 & 7.7 & 3.8 & 8.9 & 0.0 & 1.8 & 10.2 \\
\hline
\end{tabular}

Note: $-=$ no data . 